\title{
Dynamics of fitness distributions in the presence of a phenotypic optimum: an integro-differential approach* $^{*}$
}

\author{
M.-E. Gil ${ }^{\mathrm{a}, \mathrm{b}}$, F. Hamel ${ }^{\mathrm{a}}$, G. Martin ${ }^{\mathrm{c}}$ and L. Roques ${ }^{\mathrm{b}}$ \\ a Aix Marseille Univ, CNRS, Centrale Marseille, I2M, Marseille, France \\ b BioSP, INRA, 84914, Avignon, France \\ c ISEM (UMR 5554), CNRS, 34095, Montpellier, France
}

October 16, 2018

\begin{abstract}
We propose an integro-differential description of the dynamics of the fitness distribution in an asexual population under mutation and selection, in the presence of a phenotype optimum. Due to the presence of this optimum, the distribution of mutation effects on fitness depends on the parent's fitness, leading to a non-standard equation with "contextdependent" mutation kernels.

Under general assumptions on the mutation kernels, which encompass the standard $n$-dimensional Gaussian Fisher's geometrical model (FGM), we prove that the equation admits a unique time-global solution. Furthermore, we derive a nonlocal nonlinear transport equation satisfied by the cumulant generating function of the fitness distribution. As this equation is the same as the equation derived by Martin and Roques (2016) while studying stochastic Wright-Fisher-type models, this shows that the solution of the main integro-differential equation can be interpreted as the expected distribution of fitness corresponding to this type of microscopic models, in a deterministic limit. Additionally, we give simple sufficient conditions for the existence/non-existence of a concentration phenomenon at the optimal fitness value, i.e, of a Dirac mass at the optimum in the stationary fitness distribution. We show how it determines a phase transition, as mutation rates increase, in the value of the equilibrium mean fitness at mutation-selection balance. In the particular case of the FGM, consistently with previous studies based on other formalisms (Waxman and Peck, 1998, 2006), the condition for the existence of the concentration phenomenon simply requires that the dimension $n$ of the phenotype space be larger than or equal to 3 and the mutation rate $U$ be smaller than some explicit threshold.

The accuracy of these deterministic approximations are further checked by stochastic individual-based simulations.
\end{abstract}

*This work has been carried out in the framework of Archimède Labex of Aix-Marseille University. The project leading to this publication has received funding from Excellence Initiative of Aix-Marseille University A*MIDEX, a French "Investissements d'Avenir" programme, from the European Research Council under the European Union's Seventh Framework Programme (FP/2007-2013) ERC Grant Agreement n. 321186 - ReaDi Reaction-Diffusion Equations, Propagation and Modelling and from the ANR NONLOCAL project (ANR-14CE25-0013). 


\section{Introduction}

Understanding the complex interplay between mutation and selection in asexuals is a central issue of evolutionary biology. Recently, several modeling approaches have been proposed, to describe the evolution of a population under the effects of these two forces. Most of these modeling approaches assume that the fitness of the individuals depends on a single quantitative trait (e.g., $[2,23,36])$. Other approaches, as in this study, directly focus on the dynamics of fitness distributions (e.g., [1, 29]).

A rich literature (reviewed in [33]) has also focused on the interplay between drift selection and mutation in asexuals. These theories focus mainly on the expected mean fitness of the population as it reaches a stationary regime, using models that directly focus on fitness distributions. A few also considered transient behaviors of these models (e.g. [31, 35]), which can be seen as an approximation for the expected distribution of fitness, over time (discussed in $[29])$.

As fitness is a concept of fundamental importance to our study, we begin with an intuitive definition of this concept. In an asexual population made of $K$ genotypes, we say that the genotype $i$ has absolute Malthusian fitness $m_{i}$ if the abundance $N_{i}(t)$ of the genotype at time $t$ satisfies $N_{i}^{\prime}(t)=m_{i} N_{i}(t)$. Summing over all the indexes $i=1, \ldots, K$, we observe that the total population size satisfies $N^{\prime}(t)=\bar{m}(t) N(t)$, with $\bar{m}(t)$ the mean fitness in the population at time $t$. If we focus on the frequency $p_{i}(t)=N_{i}(t) / N(t)$, we get $p_{i}^{\prime}(t)=p_{i}(t)\left(m_{i}-\bar{m}(t)\right)$.

In this study, following the standard framework of Wright-Fisher or Moran models [25], we assume a constant population size $N$ and a continuum of fitness classes $m \in \mathbb{R}$. In this case, only relative Malthusian fitness matters. If we first neglect the effects of mutations, the changes in genotype frequencies due to selection are determined by their relative fitness $m$ through the expression (see, e.g., [35]):

$$
\partial_{t} p(t, m)=p(t, m)(m-\bar{m}(t)),
$$

where $m \in \mathbb{R}$ is the relative Malthusian fitness, $p(t, m)$ the distribution of fitness at time $t$, and $\bar{m}: \mathbb{R}_{+} \rightarrow \mathbb{R}$ is the mean fitness, defined for any $t \in \mathbb{R}_{+}$by

$$
\bar{m}(t)=\int_{\mathbb{R}} m p(t, m) d m .
$$

As we can observe in (1), contrarily to other traits, fitness determines its own evolution. We can also note that the notion of relative fitness is defined by (1) up to an additive constant.

A second key step is to describe the distribution of mutation effects on fitness (or DFE, in short). Most mathematical models of asexual evolution (e.g. all those reviewed in [33]) neglect the dependence of the DFE on the fitness of the parent, as discussed e.g. in [18]. Some also ignore deleterious mutations and focus only on the contribution from beneficial ones [33]. However, accumulating data from evolution experiments, often in asexual microbes, has shown that fitness epistasis is pervasive, i.e. that the fitness effects of mutations (individual effects or full distributions) depend on the genetic background in which they arise (e.g. [9, 18, 24]). This has lead to the idea that epistasis, once taken into account, might actually make evolution predictable in spite of stochastic effects due to drift and mutation, at least in large populations and at the fitness level $[18,27,29]$. This may explain why observed fitness trajectories, in large asexual populations, appear relatively repeatable (across biological replicates), in spite of non-repeatable patterns at the sequence level (e.g. [24]). This conjecture partly motivates the present study of phenotypic adaptation as a deterministic dynamical system. 


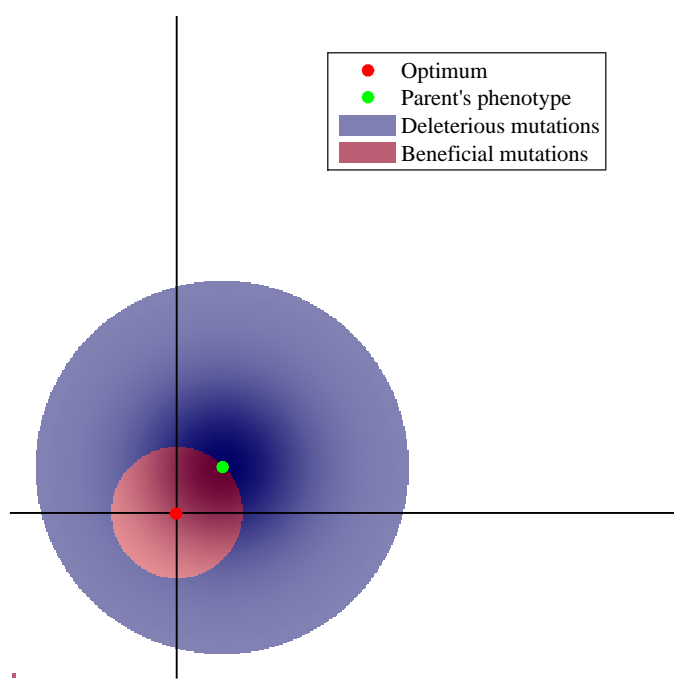

(a)

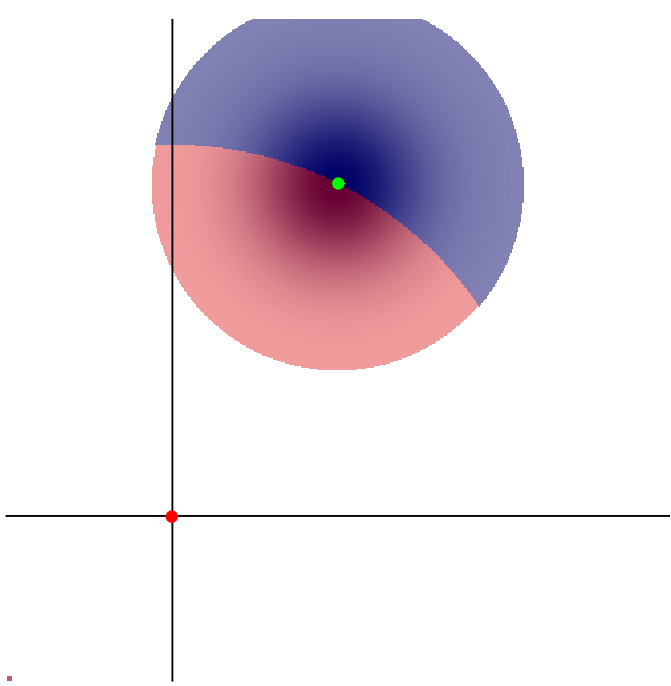

(b)

Figure 1: Proportions of beneficial and deleterious mutations, in a two-dimensional phenotypic space with a phenotypic optimum. In panel (b) the parent's phenotype is farther from the optimum than in panel (a), so that beneficial mutations are more frequent and of larger effect on average in (b).

One option to implement epistasis into fitness dynamics models is by using Fisher's Geometrical Model (FGM) with a single optimum, where a complex form of epistasis arises naturally. The FGM has been shown to lead to fairly realistic DFEs (e.g., [28]) in their shape, dependence on the environment or epistatic pattern (reviewed in [34]). It is a phenotype-fitness landscape model: it assumes $n$-dimensional (breeding values for) phenotype, described by vectors $\mathbf{z} \in \mathbb{R}^{n}$. The connection between phenotypes and relative Malthusian fitness is made through a quadratic function $m=-\|\mathbf{z}\|^{2} / 2$, with $\|\cdot\|$ the Euclidian norm in $\mathbb{R}^{n}$. A standard way of describing the effects of mutations on phenotypes is to assume that, given a parent with phenotype $\mathbf{z}$, the mutant offspring has a phenotype $\mathbf{z}+d \mathbf{z}$, where $d \mathbf{z}$ follows an $n$-dimensional isotropic Gaussian distribution with variance $\lambda>0$ at each trait (Gaussian FGM). In this case, even if the distribution of mutation effects on phenotype is independent of the parent phenotype, the DFE on fitness does depends on the parent fitness (because of the non-linear relationship between $\mathbf{z}$ and $m$ ). This is illustrated in Fig. 1. These effects can be summarized by a mutation kernel $J_{y}$ : given a parent with fitness $y$, the mutant offsprings have fitness $y+s$, with $s$ a random variable with density $J_{y}$. We say that this DFE is context-dependent because of its dependence on $y$. In spite of this complication, fitness still entirely determines its own evolution, in the FGM under selection and mutation, because epistasis is mediated by fitness alone.

Combining the equation (1) with the general assumption of a DFE that depends on background fitness, we obtain the following integro-differential equation:

$$
\partial_{t} p(t, m)=U\left(J_{y} \circledast p-p\right)(t, m)+p(t, m)(m-\bar{m}(t)), t \geq 0, m \in \mathbb{R},
$$

where $U>0$ is a given constant corresponding to the mutation rate and $J_{y} \circledast p$ is defined, for 
any $(t, m) \in \mathbb{R}_{+} \times \mathbb{R}$, by

$$
\left(J_{y} \circledast p-p\right)(t, m)=\int_{\mathbb{R}} J_{y}(m-y) p(t, y) d y-p(t, m) .
$$

This equation corresponds to a generalization of the equations studied in [17], where $J_{y}=J$ was supposed to be independent of the fitness $y$ of the parent, leading to a standard convolution product instead of the operator $\circledast$. These previous approaches with context-independent DFEs ignore all forms of epistasis, including, for example, that generated by the presence of a fitness optimum. Similarly, other approaches where the mutation effects are modeled with a diffusion approximation, i.e., when $U\left(J_{y} \circledast p-p\right)(t, m)$ is replaced by $D \partial_{m m} p(t, m)$ for some $D>0$ also ignore epistasis $([1,35])$. In these two cases (as soon as the support of $J$ intersects $\mathbb{R}_{+}$, i.e., in the presence of beneficial mutations), the mean fitness $\bar{m}(t)$ converges to $+\infty$ at large times, in a non-realistic (superlinear) way, see [31] for a discussion on this aspect.

A closely related work has been proposed in [29]. Their study focuses on a stochastic individual-based Wright-Fisher model combined with the FGM for the description of mutation effects on fitness. Based on formal computations, they derived nonlocal nonlinear transport equations satisfied by some generating function of the fitness distribution. Under some assumptions corresponding to a diffusion approximation of the mutation effects on phenotype, approximate linear transport equations arise that can be solved analytically, allowing to infer the corresponding dynamics of the multivariate phenotype and fitness distribution. Another related work has been developed by [2]. Their approach describes the dynamics of the distribution of a 1-dimensional trait $x$, with corresponding fitness value $-x^{2}$, i.e., in the presence of a fitness optimum at $x=0$. They managed, under a diffusion approximation for the mutation effects on phenotypes, to give a full analytical description of the dynamics of the trait distribution.

From a mathematical perspective, the equation (3) combines several difficulties, compared to standard reaction-diffusion equations $\partial_{t} u=D \partial_{x x} u+f(u)$ with local diffusion and local reaction terms. The mutation term $U\left(J_{y} \circledast p-p\right)$ is nonlocal, has no regularizing properties, and is not a standard convolution product. The selection term (1) is also nonlocal due to the term $\bar{m}(t)$. Equations of the type $\partial_{t} u=(J \star u-u)+f(u)$, with $\star$ the convolution product and a local reaction term $f(u)$, have been extensively studied, especially regarding the existence/nonexistence of traveling wave solutions and other spreading properties $[3,7,11,14$, $15,32,39,40,41]$. In the work [17] that was mentioned above, we considered nonlocal reaction terms of the form (1), but again with a standard convolution product. In the recent work [5], a reaction-diffusion equation with a general reaction term of the form $f(x)-\int_{\mathbb{R}} f(y) u(t, y) d y$ with $f(x) \rightarrow-\infty$ as $|x| \rightarrow \infty$, generalizing the results in [2], has also been thoroughly studied. Reaction-diffusion equations with other types of nonlocal reaction terms have also been investigated in recent works $[4,6,12,13,16,19,21]$. Lastly, we mention that operators of the type $J_{y} \circledast p-p$ or more generally of the type $\int_{\mathbb{R}} K(m, y) p(t, y) d y-p(t, m)$ have also been considered in $[8,10]$ with an emphasis on the study of the stationary states. Moreover, the existence of stationary states and travelling waves for equations of the form $\partial_{t} u=\partial_{x x} u+$ $\mu(M \circledast u-u)+f(u)$, with $f(u)$ a nonlocal reaction term, has been studied in [20].

The paper is organized as follows. In Section 2, we detail all the assumptions on the initial condition $p(0, \cdot)=p_{0}$ and on the mutation kernels $J_{y}$. In Section 3, we present our main results on equation (3). In particular, we derive some a priori estimates on the solutions (Section 3.1); we state an existence and uniqueness result (Section 3.2); we connect this equation with the formal theory developed in [29] for asexual models with selection and epistatic mutation 
(Section 3.3); we give a qualitative description of the stationary states of (3), and we apply these results to the particular case of the Gaussian FGM (Section 3.4). In Section 4, we present some numerical computations of the solutions of (3) under the assumptions of the Gaussian FGM, and we compare the corresponding distributions with observed distributions from individual-based simulations of the Wright-Fisher model. Lastly, we discuss our results in Section 5. Our results are proved in Section 6.

\section{Assumptions}

Initial condition. We assume throughout this paper that the initial distribution of fitness $p_{0} \in L^{\infty}(\mathbb{R}) \cap L^{1}(\mathbb{R})$ is a probability density function, that is,

$$
p_{0}(m) \geq 0 \text { for all } m \in \mathbb{R} \text { and } \int_{\mathbb{R}} p_{0}(m) d m=1 .
$$

Additionally, we assume that $p_{0}$ satisfies

$$
\lim _{m \rightarrow-\infty} p_{0}(m) \mathrm{e}^{\alpha|m|}=0 \text { for all } \alpha>0 .
$$

As mentioned in the introduction, we assume that there is a fitness optimum $m^{\star}$ and, without loss of generality as we work with relative fitnesses, we can assume that $m^{\star}=0$. Thus, at $t=0$, all fitnesses must be less than or equal to 0 . This means that the initial distribution $p_{0}$ is such that:

$$
\operatorname{supp} p_{0} \subset \mathbb{R}_{-}:=(-\infty, 0]
$$

General mutation kernels. We begin with the general assumptions that we use for our existence and uniqueness result. For each fitness $y \in \mathbb{R}_{-}$, we assume that $J_{y} \in L^{1}(\mathbb{R})$ is a probability density function:

$$
J_{y} \geq 0 \text { in } \mathbb{R} \text { and } \int_{\mathbb{R}} J_{y}(s) d s=1
$$

For mathematical convenience, we also set $J_{y}=0$ in $\mathbb{R}$ for each $y \in(0,+\infty)$ and we assume that, for each $m \in \mathbb{R}$, the function $y \mapsto J_{y}(m-y)$ is measurable in $\mathbb{R}$ and finite almost everywhere (a.e. for short) in $\mathbb{R}$. As 0 is the fitness of the optimum, mutant offspring from any parent with fitness $y$ cannot have a fitness larger than 0 . It follows that, for each $y \leq 0$, $-y$ is an upper bound of the support of the mutation kernel $J_{y}$, that is,

$$
J_{y}=0 \text { a.e. in }(-y,+\infty) .
$$

When $y=0$, the parent has the optimal fitness and all mutations lead to fitnesses $m \leq 0$. This is consistent with the assumption (9): the kernel $J_{0}$ is supported in $(-\infty, 0]$, and therefore leads to deleterious mutations only.

For technical reasons, we may also assume that the kernels $\left(J_{y}\right)_{y \leq 0}$ are uniformly bounded in $\mathbb{R}$ by a nonnegative function $\bar{J} \in L^{1}(\mathbb{R})$ which decays faster than any exponential function at $-\infty$, in the sense that

$$
J_{y} \leq \bar{J} \text { a.e. in } \mathbb{R} \text { for all } y \leq 0 \text {, and } \int_{-\infty}^{0} \bar{J}(m) \mathrm{e}^{\alpha|m|} d m<+\infty \text { for all } \alpha \geq 0 .
$$




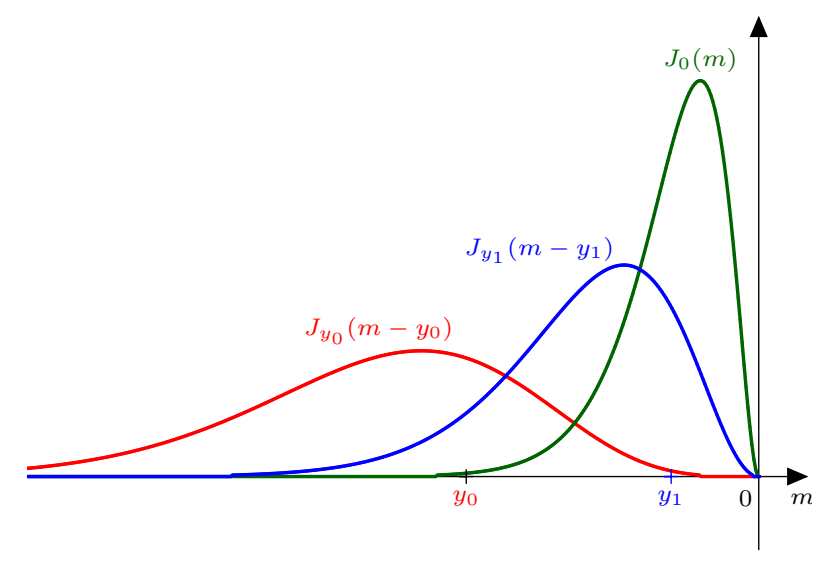

Figure 2: Mutation kernels $J_{y}$ corresponding to the Gaussian Fisher's geometric model, with phenotype dimension $n=6$ and variance $\lambda=1$, for parents with fitnesses $y_{0}<0, y_{1}<0$ and 0 .

Kernels with log-linear moment generating function. In order to connect our results with the theory described in [29], and to derive additional properties on the stationary states of (3), we require some additional assumptions on the kernels $J_{y}$. We assume here that these kernels have log-linear moment generating function in the sense that

$$
\int_{-\infty}^{-y} J_{y}(m) \mathrm{e}^{m z} d m=M_{\star}(z) \mathrm{e}^{\omega(z) y} \text { for all } y \leq 0 \text { and } z \geq 0,
$$

where

$$
M_{\star}(z)=\int_{-\infty}^{0} J_{0}(s) \mathrm{e}^{s z} d s
$$

is the moment generating function of the mutation kernel at the optimal fitness and $\omega \in \mathcal{C}^{1}\left(\mathbb{R}_{+}\right)$ satisfies

$$
\omega \not \equiv 0 \text { and } \omega^{\prime}(0) \leq 0 .
$$

The assumptions (11)-(12) are satisfied in several standard models (discussed in [29]). For example, in the Gaussian Fisher's Geometric model (FGM) mentioned in Section 1, we have [29]:

$$
\omega(z)=-\frac{\lambda z^{2}}{1+\lambda z} \text { and } M_{\star}(z)=\frac{1}{(1+\lambda z)^{n / 2}},
$$

with $\lambda>0$ being the variance of mutation effects per phenotypic trait, and $n$ the dimension of the phenotype space. The mutation kernels $J_{y}$ are uniquely defined by their moment generating function $z \mapsto M_{\star}(z) \mathrm{e}^{\omega(z) y}$ and, in the Gaussian FGM, their probability density function (pdf) takes explicit form:

$$
J_{y}(m)=\frac{2}{\lambda} f_{\chi_{n}^{2}\left(\frac{-2 y}{\lambda}\right)}\left(-\frac{2(m+y)}{\lambda}\right)
$$

for all $y \leq 0$ and $m \leq-y$, where $f_{\chi_{n}^{2}\left(\frac{-2 y}{\lambda}\right)}$ denotes the pdf of the noncentral chi-square distribution with $n$ degrees of freedom and noncentrality $-2 y / \lambda$; see Fig. 2 for an illustration. Notice that these kernels also fulfill the assumptions (8)-(10). 
The assumption $\omega \not \equiv 0$ in (12) simply means that the kernels $J_{y}$ do depend on the fitness of the parents. The assumption $\omega^{\prime}(0) \leq 0$ in (12) can be interpreted as follows. Using (11), we get that:

$$
\mathcal{C}_{y}(z)=\mathcal{C}_{\star}(z)+\omega(z) y
$$

for all $y \leq 0$ and $z \geq 0$, where

$$
\mathcal{C}_{y}(z)=\ln \left(\int_{-\infty}^{-y} J_{y}(m) \mathrm{e}^{m z} d m\right) \text { and } \mathcal{C}_{\star}(z)=\ln M_{\star}(z)=\ln \left(\int_{-\infty}^{0} J_{0}(m) \mathrm{e}^{m z} d m\right)
$$

are the cumulant generating functions of $J_{y}$ and $J_{0}$ respectively. Then, from assumption (10), the functions $\mathcal{C}_{y}$ and $\mathcal{C}_{*}=\mathcal{C}_{0}$ are of class $C^{\infty}\left(\mathbb{R}_{+}\right)$and they satisfy

$$
\mathcal{C}_{y}^{\prime}(0)=\mathcal{C}_{\star}^{\prime}(0)+\omega^{\prime}(0) y \text { for all } y \leq 0 .
$$

It follows that the mean effect of mutations on fitness $y$, namely $\mathcal{C}_{y}^{\prime}(0)=\int_{-\infty}^{-y} m J_{y}(m) d m$, is a non-increasing function of the fitness of the parent $y: \frac{d}{d y} \mathcal{C}_{y}^{\prime}(0)=\omega^{\prime}(0) \leq 0$. In the special case of the FGM (Fig. 1), the parent fitness does not affect the expected fitness effect of mutations $\left(\omega^{\prime}(0)=0\right)$.

\section{Main results}

\subsection{Support of the solution}

Under the assumption (9) on the mutation kernel, it is natural to expect that the upper bound of the support of the solution $p$ of (3) remains below the fitness optimum 0 . As stated by the proposition below, this is true even without assumptions (6) and (10) on the exponential decay of $p_{0}$ and $J_{y}$ at $-\infty$.

Proposition 3.1 Assume that $p_{0} \in L^{\infty}(\mathbb{R}) \cap L^{1}(\mathbb{R})$ satisfies assumption (7) and the mutation kernels $J_{y} \in L^{1}(\mathbb{R})$ satisfy assumptions (8) and (9). Then, for any $T \in(0,+\infty]$ and any nonnegative solution $p$ of (3) such that

$$
p \in \mathcal{C}^{1}\left([0, T), L^{\infty}(\mathbb{R}) \cap L^{1}(\mathbb{R})\right) \text { and } \bar{m} \in \mathcal{C}([0, T)),
$$

there holds

$$
\operatorname{supp} p(t, \cdot) \subset(-\infty, 0] \text { for all } t \in[0, T) \text {. }
$$

The assumptions and conclusion of Proposition 3.1 show that the integral in (2) is computed over $(-\infty, 0]$, as is the integral in $(4)$, since $J_{y}=0$ in $\mathbb{R}$ for all $y>0$. From now on, we may therefore define $\bar{m}$ and $J_{y} \circledast p$ as

$$
\bar{m}(t)=\int_{-\infty}^{0} m p(t, m) d m \text { and }\left(J_{y} \circledast p\right)(t, m)=\int_{-\infty}^{0} J_{y}(m-y) p(t, y) d y .
$$

Moreover, for every $t \in[0, T)$, the function $\left(J_{y} \circledast p\right)(t, \cdot)$ belongs to $L^{1}(\mathbb{R})$ and therefore the integral defining $\left(J_{y} \circledast p\right)(t, m)$ converges for almost every $m \in \mathbb{R}$.

Furthermore, the distribution $p(t, \cdot)$ may or not reach the fitness optimum 0 . The proposition below shows that if the kernels $J_{y}$ include some beneficial mutations for any $y<0$, in some strong sense, then the optimum is instantaneously reached. In other words, the following result gives sufficient conditions for the upper bound of the support of the solution $p(t, \cdot)$ to be equal to 0 . 
Proposition 3.2 Assume that the mutation kernels $J_{y} \in L^{1}(\mathbb{R})$ satisfy assumptions (8)-(9) and that the map

$$
m \mapsto \int_{-\infty}^{0} J_{y}(m-y) d y
$$

belongs to $L^{\infty}(\mathbb{R})$. For any $y<0$, set

$$
S_{y}=\sup \left\{s>0 \text { s.t. } J_{y}>0 \text { a.e. in }(0, s)\right\} \leq-y,
$$

and assume that

$$
S_{y}>0 \text { for all } y<0 \text { and the map } y \mapsto S_{y} \text { is continuous in }(-\infty, 0) \text {. }
$$

Assume that $p_{0} \in L^{\infty}(\mathbb{R}) \cap L^{1}(\mathbb{R})$ satisfies assumptions (5) and (7) and assume that, for some $T \in(0,+\infty]$, equation $(3)$ admits a nonnegative solution $p \in \mathcal{C}^{1}\left([0, T), L^{\infty}(\mathbb{R}) \cap L^{1}(\mathbb{R})\right)$ such that $\bar{m} \in \mathcal{C}([0, T))$ and $t \mapsto \tilde{p}(t, \cdot) \in \mathcal{C}^{1}\left([0, T), L^{\infty}(\mathbb{R})\right)$ with $\tilde{p}(t, m)=\mathrm{e}^{-t m} p(t, m)$. Then

$$
\sup \{\operatorname{supp} p(t, \cdot)\}=0 \text { for all } t \in(0, T) \text {. }
$$

The above result is reminiscent of the strong parabolic maximum principle in parabolic equations (see e.g. [30]): it shows that, provided that each kernel $J_{y}$ (for $y<0$ ) includes some beneficial mutations in the sense of (18), the support of the solution propagates with infinite speed, so that it instantaneously reaches the optimum $m=0$. This property may not be satisfied without the assumption (18).

\subsection{Global existence and uniqueness}

We are now in position to state our existence and uniqueness result.

Theorem 3.3 (Existence, uniqueness, exponential decay) Assume that $p_{0} \in L^{\infty}(\mathbb{R}) \cap$ $L^{1}(\mathbb{R})$ satisfies assumptions (5)-(7) and the kernels $J_{y}$ satisfy assumptions (8)-(10). Then problem (3) with initial condition $p_{0}$ admits a unique solution $p \geq 0$ such that

(i) $t \mapsto p(t, \cdot) \in \mathcal{C}^{1}\left([0,+\infty), L^{\infty}(\mathbb{R}) \cap L^{1}(\mathbb{R})\right), \bar{m} \in \mathcal{C}([0,+\infty))$, supp $p(t, \cdot) \subset(-\infty, 0]$ for all $t \in[0,+\infty)$, and

$$
\int_{\mathbb{R}} p(t, m) d m=\int_{-\infty}^{0} p(t, m) d m=1 \text { for all } t \geq 0
$$

(ii) $p$ decays faster than any exponential function as $m \rightarrow-\infty$ in the sense that, for every $\alpha>0$ and $T>0$, there is $\Gamma_{\alpha, T}>0$ such that:

$$
0 \leq p(t, m) \leq \Gamma_{\alpha, T} \mathrm{e}^{\alpha m} \text { for all } t \in[0, T] \text { and a.e. } m \in(-\infty, 0] \text {. }
$$

Lastly, the same decay property (20) is valid for $\left|\partial_{t} p(t, m)\right|$ as well.

The decay assumptions (6) and (10) on $p_{0}$ and $J_{y}$ seem purely technical in the proof. It is an interesting but still open question to prove the same type of result without these hypotheses. 


\subsection{Cumulant generating function}

Our goal here is to connect the equation (3) with the formal theory developed in [29] for WrightFisher individual-based models. In [29], the authors derived a nonlocal transport equation approximately satisfied by the expected cumulant generating function (CGF, for short) of the fitness distribution.

In the sequel, we assume that the kernels $J_{y}$ satisfy the assumptions (11)-(12), in addition to the properties (8)-(10). Under the assumptions and notations of Theorem 3.3, we consider the nonnegative solution $p \in \mathcal{C}^{1}\left([0,+\infty), L^{\infty}(\mathbb{R}) \cap L^{1}(\mathbb{R})\right)$ of $(3)$, and we define the cumulant generating function $C \in \mathcal{C}^{1}([0,+\infty) \times[0,+\infty))$ of the fitness distribution by

$$
C(t, z):=\ln \left(\int_{\mathbb{R}} p(t, m) \mathrm{e}^{m z} d m\right)=\ln \left(\int_{-\infty}^{0} p(t, m) \mathrm{e}^{m z} d m\right),
$$

for $t \geq 0$ and $z \geq 0$. Notice that, from Theorem 3.3, the map $t \mapsto C(t, \cdot)$ is actually of class $\mathcal{C}^{1}\left([0,+\infty), C^{\infty}([0,+\infty))\right)$. Notice also that the quantity $C(t, z)$ could be defined for all $t \geq 0$ and all $z \in \mathbb{R}$ due to the decay properties (20). The CGF is a very useful tool to analyze the properties of a distribution. In particular, it is easily seen that

$$
\partial_{z} C(t, 0)=\int_{-\infty}^{0} m p(t, m) d m
$$

is the mean fitness $\bar{m}(t)$ at a time $t \geq 0$ and

$$
\partial_{z z} C(t, 0)=\int_{-\infty}^{0} m^{2} p(t, m) d m-(\bar{m}(t))^{2}
$$

is the variance in fitness within the population.

We now derive the equation satisfied by $C$. For any given $t \geq 0$ and $z \geq 0$, by multiplying equation (3) by $\mathrm{e}^{m z}$ and integrating over $(-\infty, 0]$ with respect to $m$ (all integrals below converge due to the decay properties of $p(t, \cdot)$ and $\partial_{t} p(t, \cdot)$ given in Theorem 3.3), we obtain:

$$
\begin{aligned}
\int_{-\infty}^{0} \mathrm{e}^{m z} \partial_{t} p(t, m) d m= & U \int_{-\infty}^{0} \mathrm{e}^{m z}\left(\int_{-\infty}^{0} J_{y}(m-y) p(t, y) d y-p(t, m)\right) d m \\
& +\int_{-\infty}^{0} m \mathrm{e}^{m z} p(t, m) d m-\bar{m}(t) \int_{-\infty}^{0} \mathrm{e}^{m z} p(t, m) d m .
\end{aligned}
$$

Then, using the assumption (11), we get that:

$$
\begin{aligned}
\int_{-\infty}^{0} \mathrm{e}^{m z} \int_{-\infty}^{0} J_{y}(m-y) p(t, y) d y d m & =\int_{-\infty}^{0}\left(\int_{-\infty}^{-y} J_{y}(s) \mathrm{e}^{s z} d s\right) p(t, y) \mathrm{e}^{y z} d y \\
& =M_{\star}(z) \int_{-\infty}^{0} \mathrm{e}^{y(z+\omega(z))} p(t, y) d y .
\end{aligned}
$$

Dividing the equality (22) by $\int_{-\infty}^{0} \mathrm{e}^{m z} p(t, m) d m$ and using Lebesgue's dominated convergence theorem, we then get the following proposition. In the statement, we point out that $z+\omega(z) \geq 0$ for all $z \geq 0$, as follows from the assumptions (8), (9) and (11), see (71) and Appendix B below. 
Proposition 3.4 The function $C \in \mathcal{C}^{1}([0,+\infty) \times[0,+\infty))$ is a classical solution of the following nonlocal equation

$$
\left\{\begin{aligned}
\partial_{t} C(t, z) & =\partial_{z} C(t, z)-\partial_{z} C(t, 0)+U\left(\mathrm{e}^{C(t, z+\omega(z))-C(t, z)} M_{\star}(z)-1\right), & & t \geq 0, z \geq 0, \\
C(0, z) & =C_{0}(z), & & z \geq 0, \\
C(t, 0) & =0, & & t \geq 0,
\end{aligned}\right.
$$

with initial condition

$$
C_{0}(z)=\ln \left(\int_{-\infty}^{0} p_{0}(m) \mathrm{e}^{m z} d m\right)
$$

We obtain the same equation as equation (7) in [29]. The consequences of this result are twofold. First, it shows that the solution $p(t, m)$ of $(3)$ can be interpreted as the expected (expectation among replicate populations) distribution of fitness corresponding to WrightFisher-type individual-based models, in a deterministic limit. Second, it gives a rigorous mathematical basis to the statements in [29], which were based on formal computations. In particular, it shows that the solution of (23) (provided that it is unique) is truly a cumulant generating function, in the sense that it satisfies (21), with the following immediate consequences: (i) $C(t, \cdot)$ is convex (from Theorem 3.3 and the Cauchy-Schwarz inequality, see (80) below), (ii) $C(t, \cdot)$ is a non-increasing function. These two properties were conjectured without proof in [29].

\subsection{Stationary states}

This section is devoted to the study of the stationary states of equation (3). Namely, we focus on weak solutions $p_{\infty}$ of

$$
U\left(J_{y} \circledast p_{\infty}-p_{\infty}\right)+\left(m-\bar{m}_{\infty}\right) p_{\infty}=0
$$

where

$$
\left(J_{y} \circledast p_{\infty}\right)(m)=\int_{-\infty}^{0} J_{y}(m-y) d p_{\infty}(y) \text { and } \bar{m}_{\infty}=\int_{-\infty}^{0} m d p_{\infty}(m) .
$$

In the section, in addition to the assumptions (5)-(12), we assume that the family $(p(t, \cdot))_{t \geq 0}$ converges weakly as $t \rightarrow+\infty$ to a Radon measure $p_{\infty}$ in the sense that:

$$
\lim _{t \rightarrow+\infty} \int_{\mathbb{R}} \phi(m) p(t, m) d m=\int_{\mathbb{R}} \phi(m) d p_{\infty}(m) \in \mathbb{R}
$$

for any continuous function $\phi: \mathbb{R} \rightarrow \mathbb{R}$ such that $|\phi(m)|=O(|m|)$ as $|m| \rightarrow+\infty$. It then follows from Proposition 3.1 and Theorem 3.3 that $p_{\infty}$ is nonnegative and satisfies

$$
\operatorname{supp} p_{\infty} \subset(-\infty, 0] \text { and } \int_{-\infty}^{0} d p_{\infty}(m)=1 \text {. }
$$

Notice also from (26) that $\bar{m}_{\infty}$ defined in (25) is a real number. Furthermore, the following properties hold.

Proposition 3.5 Assume that (5)-(12), (18) and (26) hold and that, for any continuous function $\phi: \mathbb{R} \rightarrow \mathbb{R}$ with compact support, the function $(-\infty, 0] \ni y \mapsto \psi(y)=\int_{\mathbb{R}} J_{y}(m-$ y) $\phi(m) d m$ is continuous. Then the measure $p_{\infty}$ satisfies

$$
\sup \left\{\operatorname{supp} p_{\infty}\right\}=0 \text { and } \bar{m}_{\infty} \geq-U \text {. }
$$


We are now interested in the existence of a positive mass at the optimal fitness (that is, $0)$. To do so, notice that the measure $p_{\infty}$ can be written as a sum of two measures:

$$
p_{\infty}=\rho \delta_{0}+(1-\rho) p^{\star}
$$

where $\rho \in[0,1], \delta_{0}$ is the Dirac measure at 0 , and $p^{\star}$ is a nonnegative measure supported in $(-\infty, 0]$ such that $p^{\star}((-\infty, 0])=1$ and $p^{\star}$ has no mass at 0 in the sense that $p^{\star}([-\varepsilon, 0]) \rightarrow 0$ as $\varepsilon \rightarrow 0$. The following result shows a relationship between the existence of a positive mass at 0 and the value of the equilibrium mean fitness $\bar{m}_{\infty}$.

Proposition 3.6 Under the same assumptions as in Proposition 3.5, the measure $p_{\infty}$, written as in (27), satisfies

$$
\rho=0 \text { or } \bar{m}_{\infty}=-U
$$

It turns out that the existence of a positive mass at 0 depends on the mutation rate $U$ and on the harmonic mean $-s_{H}^{\star}$ of the kernel at the optimal fitness $J_{0}$ defined by

$$
-s_{H}^{\star}:=\left(\int_{-\infty}^{0} \frac{J_{0}(s)}{s} d s\right)^{-1}=-\left(\int_{0}^{+\infty} M_{\star}(z) d z\right)^{-1} \in(-\infty, 0] .
$$

More precisely, the next proposition shows that $p_{\infty}$ admits a positive mass at 0 - meaning that a positive proportion $\rho$ of the population has the best possible phenotype - if $s_{H}^{\star} \neq 0$ and $U$ is not too large.

Proposition 3.7 Under the same assumptions as in Proposition 3.5, the measure $p_{\infty}$, written as in (27), is such that:

(i) if $s_{H}^{\star}=0$, then $\rho=0$; furthermore, if $s_{H}^{\star}=0$ and

$$
\lim _{z \rightarrow+\infty} z+\omega(z) \in[0,+\infty)
$$

then $\bar{m}_{\infty}>-U$;

(ii) if $s_{H}^{\star} \neq 0$, then

(a) if $U<s_{H}^{\star}$, then $\rho>0$ and $\bar{m}_{\infty}=-U$;

(b) if

$$
U>U_{c}:=\inf \left\{U \geq s_{H}^{\star}: \text { there is } z>0 \text { with } 1+U \omega(z) M_{\star}(z)=0\right\} \text {, }
$$

then $\rho=0$ and $\bar{m}_{\infty}>-U$.

These results on the conditions of existence of a positive mass $\rho>0$ at the optimum are not straightforward to interpret intuitively (see also [37, 38] for a discussion of this issue for the particular case of the FGM). If $s_{H}^{\star}=0$ (case (i)), the distribution of mutation effects $s$ from the optimal phenotype (kernel $J_{0}$ ) typically decays exponentially or faster around $s=0$. This means that the optimal phenotype produces an amount of infinitely mild deleterious mutants. These are so mildly counter-selected, relative to their optimal parent, that a population of optimal phenotypes cannot build up, even when the mutation rate is infinitely small (albeit 
non-zero). On the other hand, when $s_{H}^{\star} \neq 0$ (case (ii)), optimal phenotypes always generate non-vanishingly deleterious mutants, which tend to be counter-selected relative to their optimal parent, thus allowing the maintainance/build-up of a class of optimal phenotypes. However, even then, a large enough mutation rate leads to the erosion of this optimal class $(\rho=0)$, because selection is not strong enough to maintain it, in the face of constant mutation destroying it. This occurs a minima when $U>U_{c}$ (we point out that $U_{c}$ is finite from the assumptions made on $J_{y}$ and $\omega$ ), and cannot occur when $U<s_{H}^{\star}$.

The assumption (29) on $\omega(z)$ is more technical. As shown in Appendix A, (29) implies that, for every $y<0$, the upper bound of the support of the kernel $J_{y}$ is equal to $-y$. In other words, this means that any suboptimal parent (fitness $y<0$ ) can yield mutant offspring with the optimal phenotype (fitness 0 , mutation effect $s=-y$ ).

Consider finally Fisher's geometrical model. Namely, assume that the mutation kernels satisfy assumptions (11) and (13)-(14). Then

$$
s_{H}^{\star}=\left(\int_{0}^{+\infty} M_{\star}(z) d z\right)^{-1}=\left(\int_{0}^{+\infty} \frac{d z}{(1+\lambda z)^{n / 2}}\right)^{-1}= \begin{cases}0, & \text { if } n \leq 2, \\ \frac{\lambda(n-2)}{2}, & \text { if } n>2 .\end{cases}
$$

In other words, $s_{H}^{\star}>0$ if and only if the phenotype space has more than two dimensions. The following corollary is an immediate consequence of Proposition 3.7 under the assumptions of the Fisher's geometrical model.

Corollary 3.8 Assume (5)-(14) and (26).

(i) If $n \leq 2$, then $\rho=0$ and $\bar{m}_{\infty}>-U$;

(ii) if $n>2$, then

(a) if $U \leq s_{H}^{\star}=\lambda(n-2) / 2$, then $\rho>0$ and $\bar{m}_{\infty}=-U$;

(b) if $U>U_{c}=\frac{\lambda(n+2)^{n / 2+1}}{16(n-2)^{n / 2-1}}$, then $\rho=0$ and $\bar{m}_{\infty}>-U$.

One of the main ingredients in the proofs of the results of this section is the CGF $C_{\infty}$ of $p_{\infty}$ defined by:

$$
C_{\infty}(z)=\ln \left(\int_{\mathbb{R}} \mathrm{e}^{m z} d p_{\infty}(m)\right)=\ln \left(\int_{-\infty}^{0} \mathrm{e}^{m z} d p_{\infty}(m)\right)
$$

for $z \geq 0$. Assumption (26) shows that $C(t, z) \rightarrow C_{\infty}(z)$ as $t \rightarrow+\infty$ for all $z \geq 0$ and that $C_{\infty}$ is of class $\mathcal{C}^{1}([0,+\infty))$ and is convex in $[0,+\infty)$ as a limit of the convex functions $C(t, \cdot)$ in $[0,+\infty)$. Passing to the limit as $t \rightarrow+\infty$ in (23), we also obtain that $C_{\infty}$ is a stationary state of $(23)$ in the sense that $C_{\infty}$ is a classical solution of the following equation:

$$
C_{\infty}^{\prime}(z)-C_{\infty}^{\prime}(0)+U\left(\mathrm{e}^{C_{\infty}(z+\omega(z))-C_{\infty}(z)} M_{\star}(z)-1\right)=0, z \geq 0 .
$$

Indeed, for any $s \geq 0$, integrating $(23)$ with respect to $t$ over $[s, s+1]$ leads to:

$$
C(s+1, z)-C(s, z)=\int_{s}^{s+1}\left(\partial_{z} C(t, z)-\partial_{z} C(t, 0)+U\left(\mathrm{e}^{C(t, z+\omega(z))-C(t, z)} M_{\star}(z)-1\right)\right) d t .
$$


As the function $C$ is of class $\mathcal{C}^{1}\left(\mathbb{R}_{+} \times \mathbb{R}_{+}\right)$, the mean value theorem implies that, for each $z \geq 0$ and $s \geq 0$, there is $t_{z, s} \in[s, s+1]$ such that

$$
\begin{aligned}
\int_{s}^{s+1}\left(\partial_{z} C(t, z)-\partial_{z} C(t, 0)+U\left(\mathrm{e}^{C(t, z+\omega(z))-C(t, z)} M_{\star}(z)-1\right)\right) d t & \\
& =\partial_{z} C\left(t_{z, s}, z\right)-\partial_{z} C\left(t_{z, s}, 0\right)+U\left(\mathrm{e}^{C\left(t_{z, s}, z+\omega(z)\right)-C\left(t_{z, s}, z\right)} M_{\star}(z)-1\right) .
\end{aligned}
$$

Then, using assumption (26), we get that, for all $z \geq 0$,

$$
\lim _{t \rightarrow+\infty} \partial_{z} C(t, z)=\lim _{t \rightarrow+\infty} \frac{\int_{-\infty}^{0} m \mathrm{e}^{m z} p(t, m) d m}{\int_{-\infty}^{0} \mathrm{e}^{m z} p(t, m) d m}=\frac{\int_{-\infty}^{0} m \mathrm{e}^{m z} d p_{\infty}(m)}{\int_{-\infty}^{0} \mathrm{e}^{m z} d p_{\infty}(m)}=C_{\infty}^{\prime}(z) .
$$

Consequently, passing to the limit as $s \rightarrow+\infty$ in (34) and using $\lim _{t \rightarrow+\infty} C(t, z)=C_{\infty}(z)$, equation (33) follows. Equation (33) will be used directly in some of the proofs, rather than (24). Notice finally that

$$
C_{\infty}^{\prime}(0)=\bar{m}_{\infty}
$$

\section{Numerical computations}

The objectives of this section are (i) to check numerically the convergence of the solution of (3) towards an equilibrium; (ii) to illustrate the results of Section 3.4 on the stationary states; and (iii) to compare the distributions $p$ obeying the integro-differential equation (3) with empirical individual-based simulations given by a Wright-Fisher model. Namely, we assume that the kernels $J_{y}$ satisfy the assumptions (8)-(14). For the sake of clarity, we recall these assumptions, which can be summarized as:

$$
\left\{\begin{array}{l}
\int_{-\infty}^{-y} J_{y}(m) \mathrm{e}^{m z} d m=M_{\star}(z) \mathrm{e}^{\omega(z) y}, \\
J_{y}(m)=\frac{2}{\lambda} f_{\chi_{n}^{2}\left(\frac{-2 y}{\lambda}\right)}\left(-\frac{2(m+y)}{\lambda}\right), \omega(z)=-\frac{\lambda z^{2}}{1+\lambda z} \text { and } M_{\star}(z)=\frac{1}{(1+\lambda z)^{n / 2}}
\end{array}\right.
$$

for all $y \leq 0, m \leq-y$ and $z \geq 0$.

Description of a Wright-Fisher individual-based model. As mentioned in the introduction, we assume a constant population size $N$. Under the assumptions of the FGM, each individual $i=$ $1, \ldots, N$ is characterized by a phenotype $\mathbf{z}_{i} \in \mathbb{R}^{n}$. Its relative Malthusian fitness (exponential growth rate) is $m_{i}=-\left\|\mathbf{z}_{i}\right\|^{2} / 2$ and its corresponding Darwinian fitness is $\mathrm{e}^{m_{i}}$ (geometric growth rate, a discrete time counterpart of the Malthusian fitness). We assume non-overlapping generations of duration $\delta_{t}=1$. Each generation, selection and genetic drift are jointly simulated by the multinomial sampling of $N$ individuals from the previous generation, each with weight given by their Darwinian fitnesses. Mutations are then simulated by randomly drawing, for each individual, a Poisson number of mutations, with rate $U$. We use a classic Gaussian FGM, following e.g. [22, 26]: each single mutation has a random phenotypic effect $d \mathbf{z}$ drawn into an isotropic multivariate Gaussian distribution: $d \mathbf{z} \sim \mathcal{N}\left(0, \lambda I_{n}\right)$, where $\lambda>0$ is the mutational variance at each trait, and $I_{n}$ is the identity matrix of size $n \times n$. Multiple mutations in a single individual have additive effects on phenotype. In all our simulations, we started with a 
clonal population (all of the individuals in the population initially share the same phenotype $\mathbf{z}_{0}$ ), and assumed a population size of $N=10^{5}$ individuals.

Numerical computations. For our simulations, we considered three sets of values of the parameters $(n, \lambda, U)$, each set of value corresponding to a different scenario in Corollary 3.8. The first set of parameters is $(n, \lambda, U)=(2,1 / 30,0.05)$. It corresponds to the assumptions of the first part of Corollary 3.8 (since $n=2$ ). The second set of parameters is $(n, \lambda, U)=(6,1 / 30,0.05)$, so that $U<U_{c} \approx 0.067$, corresponding to the second case in Corollary 3.8. The third set of parameters is $(n, \lambda, U)=(6,1 / 30,0.55)$, corresponding to the last case in Corollary 3.8, with $U>U_{c} \approx 0.533$. We solved (3) in a bounded interval $[-1.2,0.05]$ (but only the values of the solutions in the interval $[-0.6,0.05]$ are displayed in the figures), using an explicit scheme in time with a time step $\delta t=0.1$. The space was discretized, with a uniform step $\delta m=0.001$. The simulations were performed using the software Matlab ${ }^{\circledR}$.

Fig. 3 depicts the dynamics of the fitness distribution obtained with the individual-based and integro-differential approaches, for the three sets of parameter values $(n, \lambda, U)$. In all cases, starting from a clonal population with initial fitness -0.2 (that is, for (3), we consider an initial condition $p_{0}$ close to the Dirac mass at -0.2$)$ we observe that the distributions $p(t, \cdot)$ obtained by solving (3) seem to converge towards a stationary distribution. The predictions of the integro-differential approach are close to the empirical distribution given by the individualbased model, from qualitative and quantitative viewpoints. Note that, for each set of parameter values, the individual-based simulation was performed only once. The results may vary among replicate simulations.

The predictions of the two approaches at large time $(t=5000)$ are depicted in Fig. 4. Consistently with the result of Corollary 3.8, the distributions in Fig. 4a satisfy $\rho=0$ and $\bar{m}_{\infty}>-U$, the distributions in Fig. 4 b satisfy $\rho>0$ and $\bar{m}_{\infty} \approx-U$, while the distributions in Fig. 4c satisfy $\rho=0$ and $\bar{m}_{\infty}>-U$. We also note in all cases a good agreement of the distributions obtained from the integro-differential model with those obtained from the individual-based approach.

\section{Discussion}

We proposed an integro-differential description of the dynamics of the fitness distribution in a population under mutation and selection, in the presence of a phenotype optimum. Under general assumptions on the mutation kernels, which encompass the standard Gaussian Fisher's Geometrical Model, we proved that the corresponding Cauchy problem (i.e., initial value problem) was "well-posed": it admits a unique time-global solution and the support of the solution remains included in $(-\infty, 0]$, consistently with the existence of a fitness optimum at $m=0$.

Furthermore, we were able to define the cumulant generating function $C(t, z)$ of the fitness distribution, and to derive a nonlocal nonlinear transport equation satisfied by $C(t, z)$. This equation is the same as the equation derived in [29] while studying stochastic Wright-Fishertype individual-based models. We illustrated the connection between equation (3) and a Wright-Fisher-type individual-based model by performing numerical simulations. Under the assumptions of the Gaussian FGM, these simulations showed that equation (3) accurately describes the individual-based dynamics of fitness distributions. Additionally, the simulations suggest that the fitness distribution converges towards a stationary state.

The equation satisfied by $C(t, z)$ leads to a precise description of these stationary states. In 

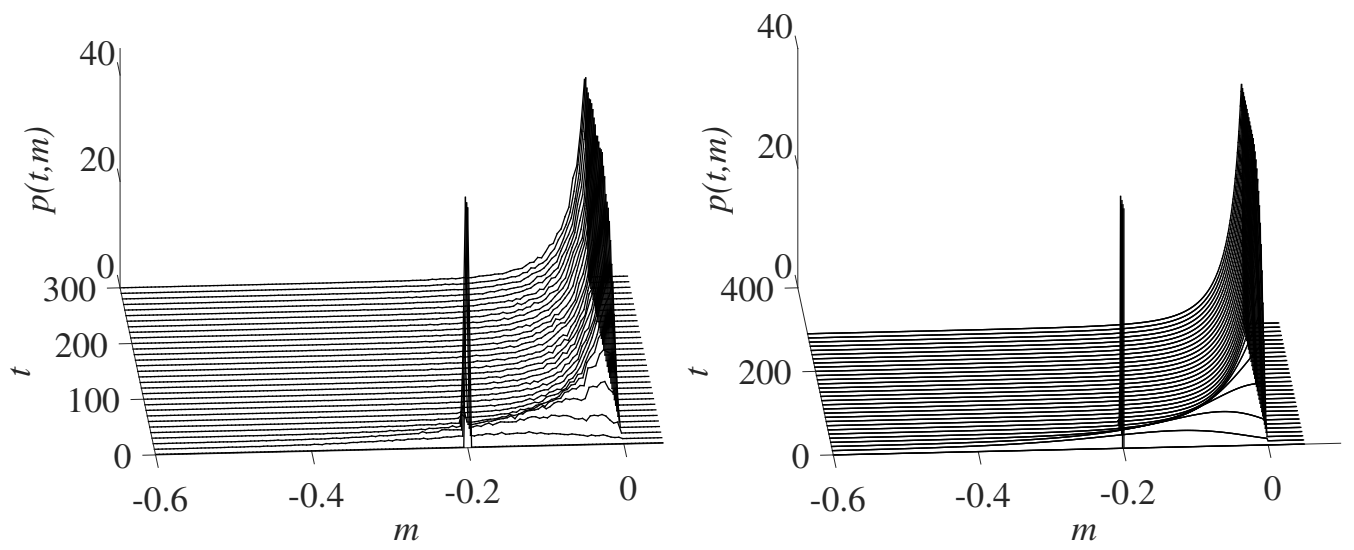

(a) $n=2, U=0.05$
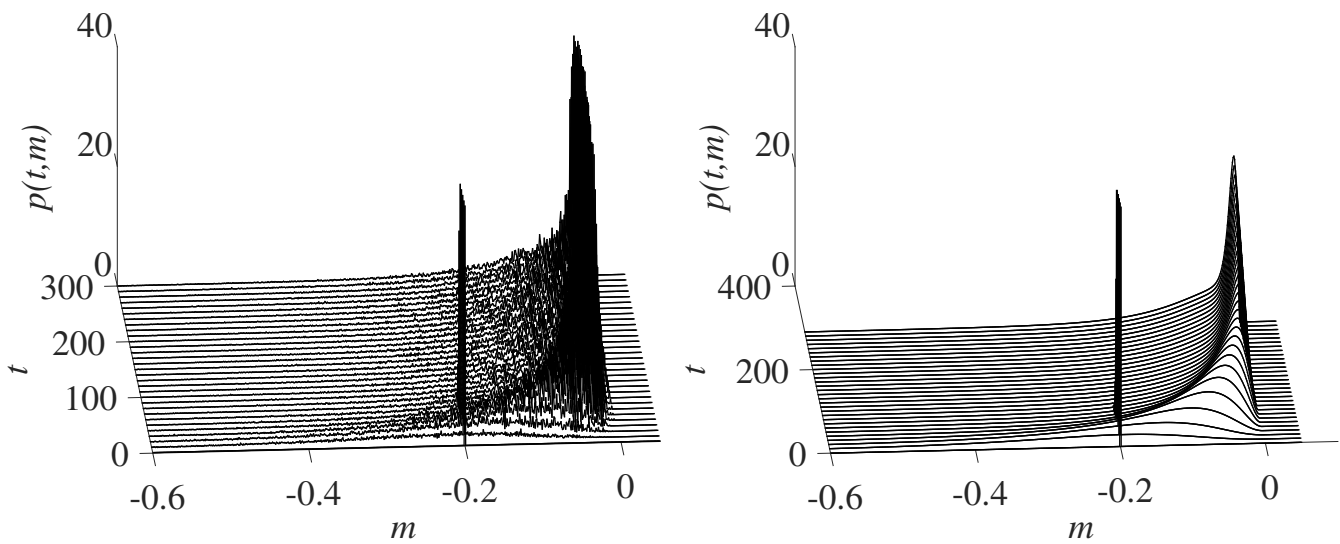

(b) $n=6, U=0.05$
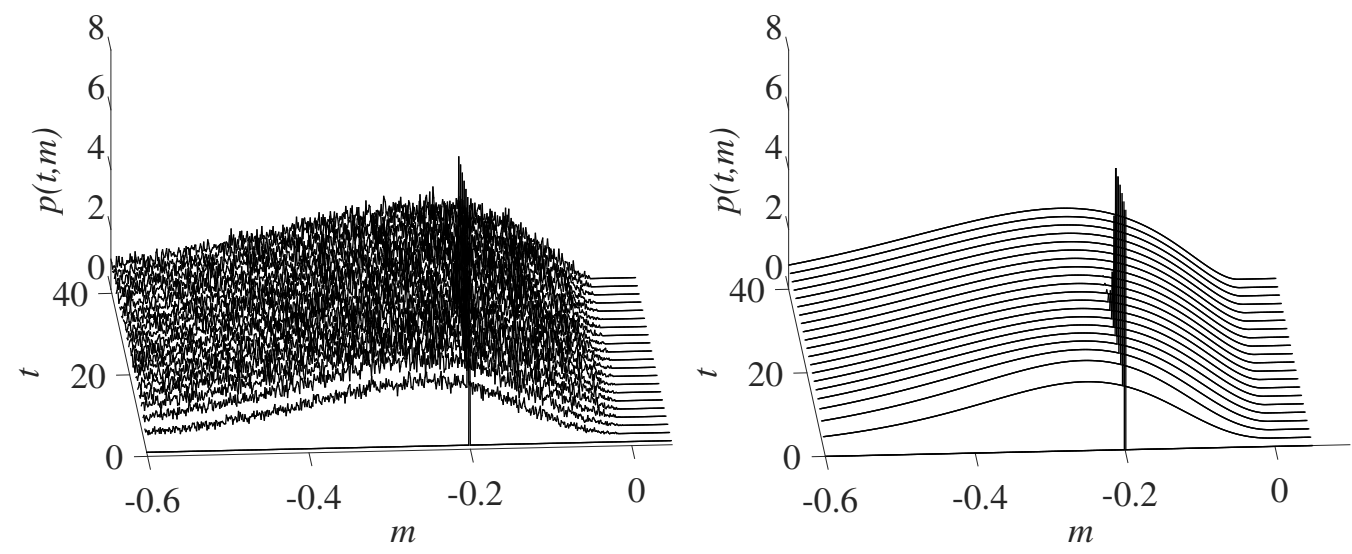

(c) $n=6, U=0.55$

Figure 3: Dynamics of the fitness distribution $p(t, m)$ : individual-based simulation with $N=$ $10^{5}$ individuals (left column) vs numerical solution of (3) with the assumption (35) (right column). In all cases, we fixed $\lambda=1 / 30$ for the mutational variance at each trait, and we assumed a clonal initial population with fitness -0.2 . 

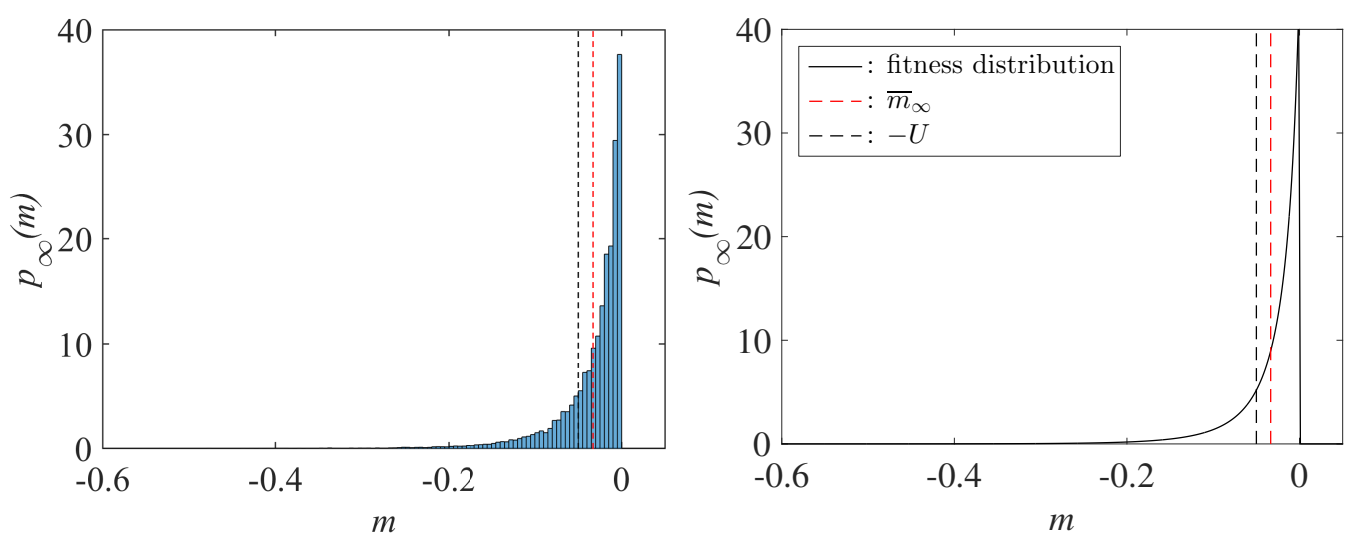

(a) $n=2, U=0.05$
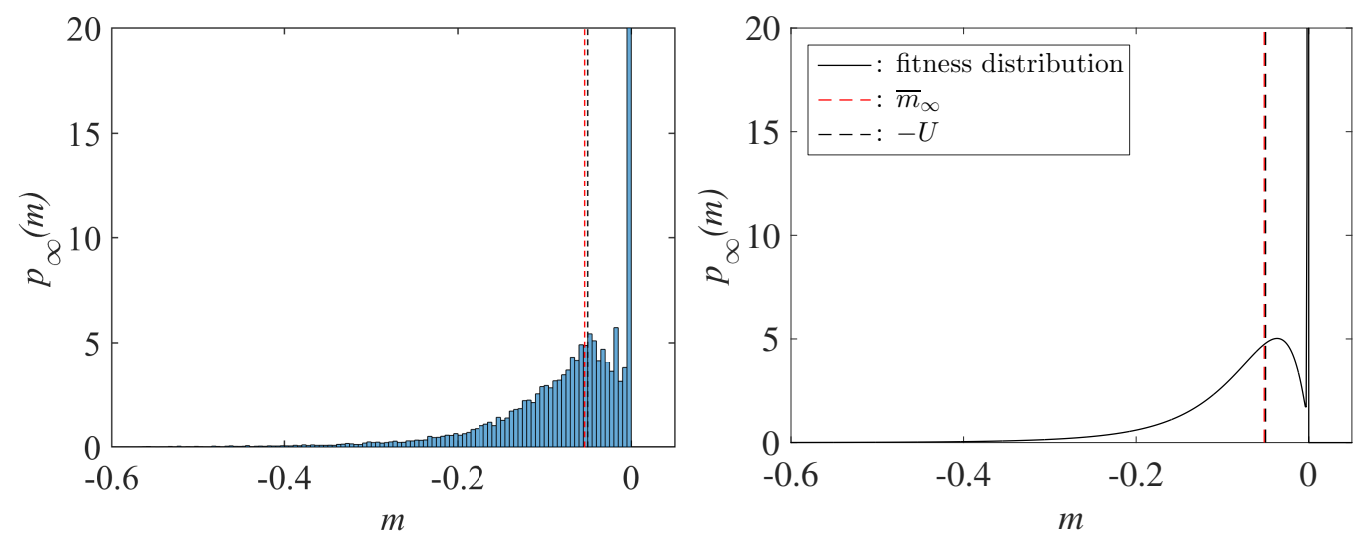

(b) $n=6, U=0.05$
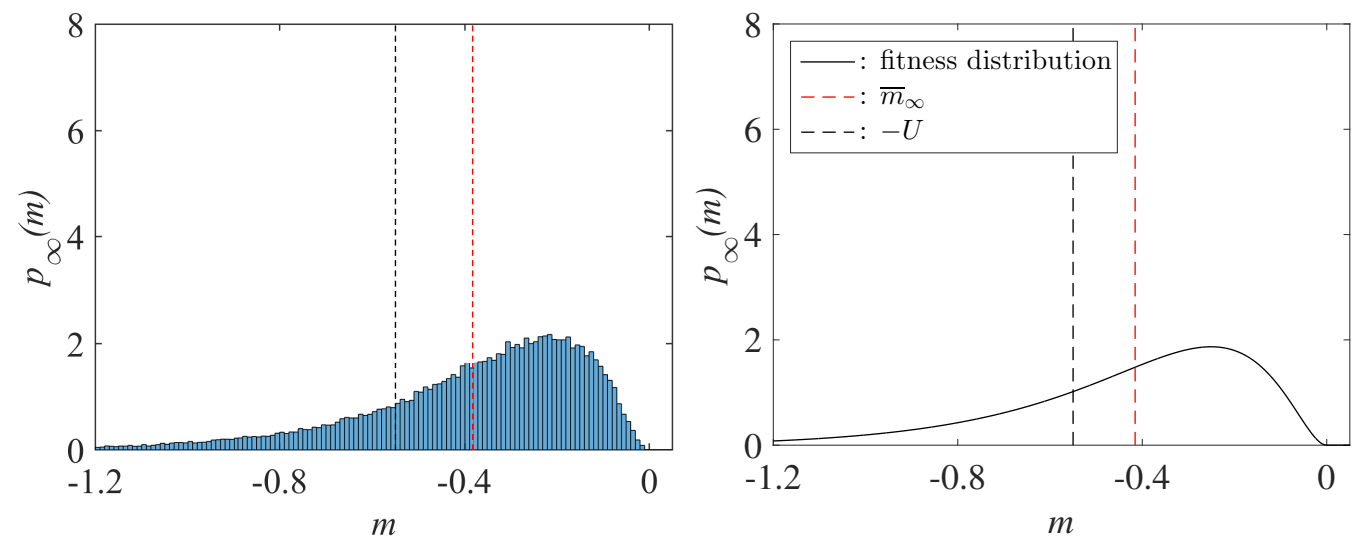

(c) $n=6, U=0.55$

Figure 4: Distribution $p(t, m)$ at large time $t=5000$, individual-based simulation (left column) vs numerical solution of (3) with the assumption (35) (right column). The parameter values are the same as in Fig. 3. 
particular, it leads to simple sufficient conditions for the existence/non-existence of a concentration phenomenon at the optimal fitness value $m=0$ (i.e, of a Dirac mass at $m=0$ in the stationary fitness distribution). Under the assumptions of the Gaussian FGM, the condition for the existence of the concentration phenomenon simply means that the dimension $n$ of the phenotype space is larger than or equal to 3 and the mutation rate $U$ is smaller than some explicit threshold. The condition on the dimension of the phenotype space is also a necessary condition: if $n \leq 2$, a positive mass at 0 can no longer exist. This is reminiscent of the results of [37] who stated that "when three or more characters are affected by each mutation, a single optimal genetic sequence may become common".

These results on the stationary states also give important clues on the equilibrium value of the mean fitness $\left(\bar{m}_{\infty}\right)$ at mutation-selection balance, called "mutation load": if a concentration phenomenon occurs at $m=0$ then, necessarily, $\bar{m}_{\infty}=-U$, where $U$ denotes the mutation rate. In the absence of concentration phenomenon, we conjecture that $\bar{m}_{\infty}>-U$ (this is true in the case of the Gaussian FGM, and in the general case under the technical assumption (29)). The determination of the exact value of $\bar{m}_{\infty}$ in this case remains a challenging open problem, although approximate treatments are proposed in [29].

The main motivation for this study was to derive rigorous mathematical results when the DFE is given by the Gaussian FGM. It is noteworthy that all of our results remain true for another standard model of context-dependent DFE: the "House of Cards" model. With this approach, given a parent with fitness $y$, the mutant offspring have fitness $s$, where $s$ is a random variable with a nonnegative fixed density $J_{H} \in L^{1}(\mathbb{R})$ supported in $(-\infty, 0]$. This means that the mutation kernels $J_{y}$ are given by

$$
J_{y}=J_{H}(y+\cdot) \text { in } \mathbb{R} \text { for every } y \leq 0 .
$$

Thus, the family $J_{y}$ satisfies the same assumption (11) as the Gaussian FGM, with this time

$$
M_{\star}(z)=\int_{-\infty}^{0} J_{H}(s) \mathrm{e}^{s z} d s \text { and } \omega(z)=-z
$$

thus, Proposition 3.7 can be applied. In this case, the assumption (29) is always fulfilled, and the occurrence of a concentration phenomenon at the optimum depends on the harmonic mean of $J_{H}$.

\section{Proofs of the main results}

This section is devoted to the proof of the main results announced in Section 3 on the solutions of $(3),(23)$ and $(33)$.

\subsection{Proof of Propositions 3.1 and 3.2 on the support of $p(t, \cdot)$}

Proof of Proposition 3.1. Assume that $p_{0} \in L^{\infty}(\mathbb{R}) \cap L^{1}(\mathbb{R})$ and the mutation kernels $J_{y} \in L^{1}(\mathbb{R})$ satisfy assumptions $(7)$-(9). Let $T \in(0,+\infty]$ and $p \in \mathcal{C}^{1}\left([0, T), L^{\infty}(\mathbb{R}) \cap L^{1}(\mathbb{R})\right)$ be a nonnegative solution of $(3)$ such that $\bar{m}$ defined by $(2)$ belongs to $\mathcal{C}([0, T))$. Let $A>0$ and denote

$$
Q_{A}(t)=\int_{0}^{A} p^{2}(t, m) d m \text { for } t \in[0, T) .
$$


The assumptions on $J_{y}$ and $p$ and Fubini's theorem imply that, for every $t \in[0, T)$, the function $\left(J_{y} \circledast p\right)(t, \cdot)$ belongs to $L^{1}(\mathbb{R})$ and that

$$
\left.\int_{a}^{b}\left(J_{y} \circledast p\right)(t, m) d m=\int_{a}^{b} \int_{\mathbb{R}} J_{y}(m-y) p t, y\right) d y d m=0
$$

for every $0 \leq a<b$, hence $\left(J_{y} \circledast p\right)(t, m)=0$ for a.e. $m>0$. Therefore, for every $t \in[0, T)$, by multiplying $(3)$ by $p(t, m)$ and integrating over $(0, A)$, we get that

$$
\frac{1}{2} \int_{0}^{A} \partial_{t}\left(p^{2}\right)(t, m) d m=-U Q_{A}(t)-\bar{m}(t) Q_{A}(t)+\int_{0}^{A} m p^{2}(t, m) d m .
$$

Since

it follows that

$$
\int_{0}^{A} m p^{2}(t, m) d m \leq A \int_{0}^{A} p^{2}(t, m) d m=A Q_{A}(t)
$$

$$
\frac{1}{2} Q_{A}^{\prime}(t) \leq(A-U-\bar{m}(t)) Q_{A}(t)
$$

for every $t \in[0, T)$. As $Q_{A}(0)=0$ according to assumption $(7)$, and since $\bar{m}(t)$ depends continuously on $t$ and $Q_{A} \geq 0$ on [0,T), Grönwall's lemma implies that $Q_{A}(t)=0$ for all $t \in[0, T)$. Since $A>0$ can be arbitrarily large, the proof of Proposition 3.1 is thereby complete.

Proof of Proposition 3.2. Assume that $p_{0}$ and the mutation kernels $J_{y} \in L^{1}(\mathbb{R})$ satisfy assumptions (5), (7)-(9) and (18). Let $T \in(0,+\infty]$ and $p \in \mathcal{C}^{1}\left([0, T), L^{\infty}(\mathbb{R}) \cap L^{1}(\mathbb{R})\right)$ be a nonnegative solution of (3) such that $\bar{m}$ defined by (2) belongs to $\mathcal{C}([0, T)$ ) and $t \mapsto \tilde{p}(t, \cdot) \in \mathcal{C}^{1}\left([0, T), L^{\infty}(\mathbb{R})\right)$ with $\tilde{p}(t, m)=\mathrm{e}^{-t m} p(t, m)$. First of all, Proposition 3.1 implies that $\operatorname{supp} p(t, \cdot) \subset(-\infty, 0]$ for all $t \in[0, T)$.

Define

$$
w(t, m)=\mathrm{e}^{-t m+\int_{0}^{t} \bar{m}(s) d s} p(t, m)
$$

for $(t, m) \in[0, T) \times \mathbb{R}$. By assumption, the function $t \mapsto w(t, \cdot)$ belongs to $\mathcal{C}^{1}\left([0, T), L^{\infty}(\mathbb{R})\right)$. As $p$ obeys (3), $w$ is a solution of the following equivalent problem:

$$
\left\{\begin{aligned}
\partial_{t} w & =U\left(J_{y}^{t} \circledast w-w\right), & & t \in[0, T), m \in \mathbb{R} \\
w(0, m) & =p_{0}(m), & & m \in \mathbb{R},
\end{aligned}\right.
$$

with

$$
J_{y}^{t}(s)=\mathrm{e}^{-t s} J_{y}(s)
$$

for $t \in[0, T), y \in \mathbb{R}$ and $s \in \mathbb{R}$. As in (3), the equality $\partial_{t} w=U\left(J_{y}^{t} \circledast w-w\right)$ is understood for every $t \in\left[0, T\right.$ ), and for almost every $m \in \mathbb{R}$ (or equivalently for almost every $m \in \mathbb{R}_{-}$by Proposition 3.1). Observe that, for every $t \in[0, T), p(t, \cdot)$ and $w(t, \cdot)$ have the same support (in particular, supp $w(t, \cdot) \subset(-\infty, 0]$ for every $t \in[0, T))$.

In order to prove that $\sup \{\operatorname{supp} w(t, \cdot)\}=0$ for all $t \in(0, T)$, we are going to construct a sub-solution $\underline{w}$ of (36). To do so, assume first, without loss of generality, that $T<+\infty$ (the conclusion in the case $T=+\infty$ would then follow from the case $T=n$ for any $n \in \mathbb{N}, n \geq 1$ ). Since $p_{0}$ is not trivial with $p_{0} \geq 0$ in $\mathbb{R}$ and since $\operatorname{supp} p_{0} \subset(-\infty, 0]$, there is $\mu<0$ such that

$$
\forall \varepsilon>0, \quad \int_{\mu-\varepsilon}^{\mu} p_{0}(m) d m>0
$$


Let now $N \in \mathbb{N}$ such that

$$
N \geq \mathrm{e}^{T(1-\mu)}>1
$$

and, for $y \leq 0$ and $s \in \mathbb{R}$, define

$$
K_{y}(s)= \begin{cases}\frac{J_{y}(s)}{N} & \text { if } s<\frac{\ln N}{T} \\ 0 & \text { if } s \geq \frac{\ln N}{T}\end{cases}
$$

Now, set

$$
\underline{w}(t, m)=\left[p_{0}(m)+U t\left(K_{y} \circledast p_{0}\right)(m)\right] \mathrm{e}^{-U t}
$$

for $(t, m) \in[0, T) \times \mathbb{R}$. Since $p_{0} \in L^{\infty}(\mathbb{R})$ and the map $m \mapsto \int_{-\infty}^{0} J_{y}(m-y) d y$ is assumed to be in $L^{\infty}(\mathbb{R})$ as well, one infers that the map $t \mapsto \underline{w}(t, \cdot)$ belongs to $\mathcal{C}^{\infty}\left([0, T), L^{\infty}(\mathbb{R})\right)$. Furthermore, since supp $p_{0} \subset(-\infty, 0]$ and $J_{y}=0$ a.e. in $(-y,+\infty)$ for all $y \leq 0$, one has $K_{y} \circledast p_{0}=0$ a.e. in $(0,+\infty)$ (as for $\left(J_{y} \circledast p\right)(t, \cdot)$ in the proof of Proposition 3.1) and

$$
\underline{w}(t, \cdot)=0 \text { a.e. in }(0,+\infty) \text {, for each } t \in[0, T) .
$$

There also holds

$$
\partial_{t} \underline{w}(t, m)=-U \underline{w}(t, m)+U \mathrm{e}^{-U t}\left(K_{y} \circledast p_{0}\right)(m)
$$

and

$$
\left(J_{y}^{t} \circledast \underline{w}\right)(t, m)=\mathrm{e}^{-U t}\left[\left(J_{y}^{t} \circledast p_{0}\right)(m)+U t\left(J_{y}^{t} \circledast\left(K_{y} \circledast p_{0}\right)\right)(m)\right],
$$

hence

$$
\begin{aligned}
\partial_{t} \underline{w}(t, m)+U \underline{w}(t, m)-U\left(J_{y}^{t} \circledast \underline{w}\right)(t, m) \\
\quad=U \mathrm{e}^{-U t}\left[\left(\left(K_{y}-J_{y}^{t}\right) \circledast p_{0}\right)(m)-U t\left(J_{y}^{t} \circledast\left(K_{y} \circledast p_{0}\right)\right)(m)\right]
\end{aligned}
$$

for $(t, m) \in[0, T) \times \mathbb{R}$. Using the nonnegativity assumptions (5) and (8), we get, for each $t \in[0, T)$ and $a<b$ in $\mathbb{R}$,

$$
\int_{a}^{b}\left(J_{y}^{t} \circledast\left(K_{y} \circledast p_{0}\right)\right)(m) d m=\int_{a}^{b} \int_{\mathbb{R}} \int_{\mathbb{R}} \mathrm{e}^{-t\left(m-y^{\prime}\right)} J_{y^{\prime}}\left(m-y^{\prime}\right) K_{y}\left(y^{\prime}-y\right) p_{0}(y) d y d y^{\prime} d m \geq 0
$$

by Fubini's theorem, hence

$$
U t\left(J_{y}^{t} \circledast\left(K_{y} \circledast p_{0}\right)\right) \geq 0 \text { a.e. in } \mathbb{R}, \text { for every } t \in[0, T) .
$$

Moreover, for each $t \in[0, T)$, one has $K_{y}(m-y)-J_{y}^{t}(m-y)=-J_{y}^{t}(m-y)=-\mathrm{e}^{-t(m-y)} J_{y}(m-y)$ if $m-y \geq(\ln N) / T$, whereas

$$
K_{y}(m-y)-J_{y}^{t}(m-y)=\underbrace{\left(\frac{1}{N}-\mathrm{e}^{-t(m-y)}\right)}_{\leq 0} J_{y}(m-y) \text { if } m-y<\frac{\ln N}{T} .
$$

As a consequence, from (8), one infers that, for every $t \in[0, T)$ and $a<b$ in $\mathbb{R}$,

$$
\int_{a}^{b}\left(\left(K_{y}-J_{y}^{t}\right) \circledast p_{0}\right)(m) d m=\int_{a}^{b} \int_{\mathbb{R}}\left(K_{y}(m-y)-J_{y}^{t}(m-y)\right) p_{0}(y) d y d m \leq 0,
$$


hence $\left(K_{y}-J_{y}^{t}\right) \circledast p_{0} \leq 0$ a.e. in $\mathbb{R}$ for every $t \in[0, T)$. Then, together with (40) and (41), we get that

$$
\partial_{t} \underline{w}(t, m) \leq U\left(J_{y}^{t} \circledast \underline{w}-\underline{w}\right)(t, m)
$$

for all $t \in[0, T)$ and for a.e. $m \in \mathbb{R}$. To sum up, $\underline{w} \in \mathcal{C}^{\infty}\left([0, T], L^{\infty}(\mathbb{R})\right)$ is a sub-solution of problem (36).

Remember also that $\underline{w}(0, \cdot)=p_{0}=w(0, \cdot)$ in $\mathbb{R}$. In order to conclude that $\underline{w}(t, \cdot) \leq w(t, \cdot)$ a.e. in $\mathbb{R}$ for every $t \in[0, T)$, we will apply the following comparison principle, whose proof in postponed in Section 7.

Lemma 6.1 (Comparison principle) Let $\tau \in(0,+\infty), \varpi \in \mathcal{C}([0, \tau])$ and $h_{1}, h_{2} \in$ $\mathcal{C}^{1}\left([0, \tau], L^{\infty}(\mathbb{R})\right)$ be such that, for every $t \in[0, \tau], h_{1}(t, \cdot)=h_{2}(t, \cdot)=0$ a.e. in $(0,+\infty)$ and

$$
\begin{aligned}
\partial_{t} h_{1}(t, m) & -U\left(\int_{\mathbb{R}} J_{y}^{t}(m-y) h_{1}(t, y) d y-h_{1}(t, m)\right)+\varpi(t) h_{1}(t, m) \\
\leq & \partial_{t} h_{2}(t, m)-U\left(\int_{\mathbb{R}} J_{y}^{t}(m-y) h_{2}(t, y) d y-h_{2}(t, m)\right)+\varpi(t) h_{2}(t, m)
\end{aligned}
$$

for a.e. $m \in \mathbb{R}_{-}$, with $J_{y}^{t}$ defined in $(37)$. Assume that $h_{1}(0, \cdot) \leq h_{2}(0, \cdot)$ a.e. in $\mathbb{R}$. Then, for every $t \in[0, \tau], h_{1}(t, \cdot) \leq h_{2}(t, \cdot)$ a.e. in $\mathbb{R}$.

Together with the fact that the map $t \mapsto w(t, \cdot)$ belongs to $\mathcal{C}^{1}\left([0, T), L^{\infty}(\mathbb{R})\right)$, Lemma 6.1 (applied with $h_{1}=\underline{w}, h_{2}=w, \varpi=0$ and every $\tau \in(0, T)$ ) yields $\underline{w}(t, \cdot) \leq w(t, \cdot)$ a.e. in $\mathbb{R}$ for every $t \in[0, T)$. Hence, for each $t \in[0, T)$, there holds

$$
p(t, m) \geq \mathrm{e}^{t m-\int_{0}^{t} \bar{m}(s) d s} \underline{w}(t, m)
$$

for a.e. $m \in \mathbb{R}$.

Let now $\sigma>0$ be such that

$$
0<\sigma<\min \left(\frac{\ln N}{2|\mu| T}, \frac{1}{2}\right)
$$

where $\mu<0$ and $N>1$ were given in (38) and (39). Let us show in this paragraph that, for every $t \in(0, T), \underline{w}(t, \cdot)>0$ in $\left[\mu, \mu+\sigma S_{\mu}\right]$, where we recall that $S_{\mu}>0$ from assumption (18). Using assumption (18) on the continuity of $(-\infty, 0) \ni y \mapsto S_{y} \in(0,-y]$ and the property (38) on $\mu$, there is $\beta \in\left(0, \sigma S_{\mu}\right]$ such that $S_{y}>2 \sigma S_{\mu}$ for all $y \in(\mu-\beta, \mu)$ and

$$
\int_{\mu-\beta}^{\mu} p_{0}(s) d s>0 .
$$

Pick any $t \in(0, T)$ and any $a<b \in\left[\mu, \mu+\sigma S_{\mu}\right]$. We have

$$
\int_{a}^{b} \frac{\mathrm{e}^{U t} \underline{w}(t, m)}{U t} d m \geq \int_{a}^{b} \int_{\mathbb{R}} K_{y}(m-y) p_{0}(y) d y d m \geq \int_{a}^{b} \int_{\mu-\beta}^{\mu} K_{y}(m-y) p_{0}(y) d y d m
$$

by Fubini's theorem and the nonnegativity of $K_{y}$ and $p_{0}$. As $0<\beta \leq \sigma S_{\mu}, \mu \leq a<b \leq \mu+\sigma S_{\mu}$ and $0<S_{\mu} \leq|\mu|$, one infers that, for every $y \in(\mu-\beta, \mu)$ and $m \in[a, b]$,

$$
0<m-y \leq 2 \sigma S_{\mu}<\min \left(S_{y}, \frac{\ln N}{T}\right) .
$$


We deduce from the definition of $S_{y}$ that

$$
\int_{a}^{b} K_{y}(m-y) d m=\int_{a}^{b} \frac{J_{y}(m-y)}{N} d m>0
$$

for all $y \in(\mu-\beta, \mu)$. Together with (44) and the nonnegativity of $p_{0}$, we get that

$$
\int_{a}^{b} \int_{\mu-\beta}^{\mu} K_{y}(m-y) p_{0}(y) d y d m>0 .
$$

As a consequence,

$$
\int_{a}^{b} \underline{w}(t, m) d m>0
$$

for all $a<b \in\left[\mu, \mu+\sigma S_{\mu}\right]$.

Pick any $t_{0}$ in $(0, T)$ and remember that $\mu<\mu+\sigma S_{\mu}<0$ since $0<\sigma<1 / 2<1$ and $0<S_{\mu} \leq-\mu=|\mu|$. It follows from (43) and the previous paragraph that

$$
\int_{a}^{b} p\left(t_{0}, m\right) d m>0
$$

for all $a<b \in\left[\mu, \mu+\sigma S_{\mu}\right]$ (and $p\left(t_{0}, \cdot\right) \geq 0$ in $\mathbb{R}$ by assumption), hence

$$
\int_{\mu+\sigma S_{\mu}-\varepsilon}^{\mu+\sigma S_{\mu}} p\left(t_{0}, m\right) d m>0
$$

for every $\varepsilon>0$. By observing that $0<S_{\mu+\sigma S_{\mu}} \leq-\left(\mu+\sigma S_{\mu}\right)<-\mu=|\mu|$ and by using the same argument as in the previous paragraph with the initial condition $p\left(t_{0}, \cdot\right)$ instead of $p_{0}$ and with $\mu+\sigma S_{\mu}$ instead of $\mu$, we get that, for every $t \in\left(t_{0}, T\right)$ and for every $a<b \in$ $\left[\mu+\sigma S_{\mu}, \mu+\sigma S_{\mu}+\sigma S_{\mu+\sigma S_{\mu}}\right]$

$$
\int_{a}^{b} \underline{w}(t, m) d m>0 .
$$

As $t_{0}$ is arbitrary in $(0, T)$ and by remembering (43), we infer that, for all $t \in(0, T)$ and $a<b \in\left[\mu+\sigma S_{\mu}, \mu+\sigma S_{\mu}+\sigma S_{\mu+\sigma S_{\mu}}\right]$,

$$
\int_{a}^{b} p(t, m) d m>0 .
$$

Consider finally the sequence $\left(\widetilde{S}_{n}\right)_{n \in \mathbb{N}}$ defined by

$$
\widetilde{S}_{0}=\mu \text { and } \widetilde{S}_{n+1}=\widetilde{S}_{n}+\sigma S_{\widetilde{S}_{n}} \text { for all } n \in \mathbb{N} .
$$

From the inequalities $0<S_{y} \leq-y$ (for all $y<0$ ) and $0<\sigma<1 / 2<1$, the sequence $\left(\widetilde{S}_{n}\right)_{n \in \mathbb{N}}$ is well-defined, increasing, and such that $\mu \leq \widetilde{S}_{n}<0$ for all $n \in \mathbb{N}$. It then converges to a limit

$$
L=\lim _{n \rightarrow+\infty} \widetilde{S}_{n}
$$

such that $\mu<L \leq 0$. If $L$ were (strictly) negative, then the continuity assumption (18) of $y \mapsto S_{y}$ in $(-\infty, 0)$ would imply that $L=L+\sigma S_{L}$, whence $S_{L}=0$, which is ruled out 
by (18). Therefore, $L=0$. On the other hand, the arguments of the previous paragraphs imply by immediate induction that (45) holds for every $t \in(0, T)$, every $n \in \mathbb{N}$ and every $a<b \in\left[\mu, \widetilde{S}_{n+1}\right]$. Hence, (45) holds for every $t \in(0, T)$ and $a<b \in[\mu, 0)$. Together with Proposition 3.1, one gets that

$$
\sup \{\operatorname{supp} p(t, \cdot)\}=0
$$

for every $t \in(0, T)$. The proof of Proposition 3.2 is thereby complete.

\subsection{Global existence: proof of Theorem 3.3}

In order to show Theorem 3.3 on the global existence of solutions of (3), the general strategy consists in applying Cauchy-Lipschitz theorem in some suitably chosen function space. To do so, we first prove the local existence, with an existence time which is quantitatively defined in terms of the kernels $\left(J_{y}\right)_{y \in \mathbb{R}_{-}}$and the initial probability density $p_{0}$.

Proposition 6.2 (Local existence) Let $\beta \geq 1$, let the kernels $J_{y}$ satisfy assumptions (8)(10) and let $p_{0} \in L^{\infty}(\mathbb{R}) \cap L^{1}(\mathbb{R})$ satisfy (5), (7) and

$$
0<K:=\operatorname{esssup}_{m \in \mathbb{R}}\left(p_{0}(m) \mathrm{e}^{-\beta m}\right)<+\infty .
$$

Let

$$
T=\min \left\{\beta,\left(U \int_{-\infty}^{0} \bar{J}(m) \mathrm{e}^{2 \beta|m|} d m+U\|\bar{J}\|_{L^{1}(\mathbb{R})}+3 K \mathrm{e}\right)^{-1}\right\}>0 .
$$

Then problem $(3)$ admits a solution $p \in \mathcal{C}^{1}\left([0, T], L^{\infty}(\mathbb{R}) \cap L^{1}(\mathbb{R})\right)$ such that $\bar{m} \in \mathcal{C}([0, T])$ and $p$ decays at least like $\mathrm{e}^{t / T+\beta m+t m}$ as $m \rightarrow-\infty$, in the sense that

$$
0 \leq p(t, m) \leq K \mathrm{e}^{t / T+(\beta+t) m} \leq K \mathrm{e}^{1+\beta m} \leq K \mathrm{e}^{1+T m} \text { for all } t \in[0, T] \text { and a.e. } m \in \mathbb{R} .
$$

Furthermore, this solution is unique.

Remark 6.3 Notice that $\operatorname{supp} p(t, \cdot) \subset \mathbb{R}_{-}$for every $t \in[0, T)$ from Proposition 3.1 and for $t=T$ too from the continuity of the map $t(\in[0, T]) \mapsto p(t, \cdot)$ in $L^{\infty}(\mathbb{R}) \cap L^{1}(\mathbb{R})$.

Proof of Proposition 6.2. Step 1: an auxiliary problem. Let $\beta, p_{0}$ and $K$ be defined as in the statement of Proposition 6.2. We first show the local existence, for some well chosen $T>0$, of a solution $v$ of the following nonlinear Cauchy problem:

$$
\left\{\begin{aligned}
\partial_{t} v(t, m) & =U\left(J_{y}^{t} \circledast v-v\right)(t, m)-\bar{m}_{v}(t) v(t, m), & & t \in[0, T], m \in \mathbb{R}, \\
v(0, m) & =p_{0}(m), & & m \in \mathbb{R},
\end{aligned}\right.
$$

with $J_{y}^{t}$ as in (37) and

$$
\bar{m}_{v}(t)=\int_{\mathbb{R}} m \mathrm{e}^{t m} v(t, m) d m .
$$

To do so, let us first introduce the Banach space

$$
L_{-}^{\infty}(\mathbb{R})=\left\{f \in L^{\infty}(\mathbb{R}) ; \operatorname{supp} f \subset \mathbb{R}_{-}\right\},
$$


pick any real number $T$ such that $0<T \leq \beta$, and consider the set

$$
\begin{aligned}
& E=\left\{f \in \mathcal { C } \left([0, T], L_{-}^{\infty}(\mathbb{R}) ; \quad f(0, \cdot)=p_{0}\right.\right. \text { and } \\
& \left.\qquad \leq f(t, m) \leq K \mathrm{e}^{t / T+\beta m} \text { for all } t \in[0, T] \text { and a.e. } m \in \mathbb{R}\right\},
\end{aligned}
$$

and denote ${ }^{1}$

$$
\|f\|_{E}=\left\|f(t, m) \mathrm{e}^{-t / T-\beta m}\right\|_{L^{\infty}([0, T] \times \mathbb{R})} .
$$

Notice that the set $E$ is not empty since the function $f$ defined by $f(t, m)=p_{0}(m)$ belongs to $E$, as $p_{0}$ satisfies (5), (7) and (46). Let us now define a map $F$ as follows:

$$
\begin{aligned}
F: E & \rightarrow \mathcal{C}^{1}\left([0, T], L_{-}^{\infty}(\mathbb{R})\right), \\
v & \mapsto h,
\end{aligned}
$$

where $h=F(v)$ is the solution of the following linear Cauchy problem

$$
\left\{\begin{aligned}
\partial_{t} h(t, m) & =U\left(\int_{\mathbb{R}} J_{y}^{t}(m-y) h(t, y) d y-h(t, m)\right)-\bar{m}_{v}(t) h(t, m), & & t \in[0, T], m \in \mathbb{R}, \\
h(0, m) & =p_{0}(m), & & m \in \mathbb{R} .
\end{aligned}\right.
$$

Notice that, since $v \in E$, the function $\bar{m}_{v}$ defined in (49) exists and belongs to $\mathcal{C}([0, T])$. Furthermore, Lemma 6.4 below states that $h$ is well defined as well.

Lemma 6.4 For any given $v \in E$ with $0<T \leq \beta$, the Cauchy problem (50) admits a unique solution $h \in \mathcal{C}^{1}\left([0, T], L_{-}^{\infty}(\mathbb{R})\right)$.

In order not to slow down the proof of Proposition 6.2, the proof of Lemma 6.4 is postponed in Section 7.

Step 2: $F$ maps $E$ to $E$ for $T>0$ small enough. Consider any function $v \in E$, for some $T \in(0, \beta]$. Since $\operatorname{supp} v(t, \cdot) \subset \mathbb{R}_{-}$for all $t \in[0, T]$ and since $0 \leq v(t, m) \leq K \mathrm{e}^{t / T+\beta m}$ for all $t \in[0, T]$ and a.e. $m \in \mathbb{R}_{-}$with $\beta \geq 1$, it follows that, for all $t \in[0, T]$,

$$
\left|\bar{m}_{v}(t)\right| \leq \int_{-\infty}^{0}|y| \mathrm{e}^{t y} v(t, y) d y \leq K \int_{-\infty}^{0}|y| \mathrm{e}^{t y} \mathrm{e}^{t / T+\beta y} d y=\frac{K \mathrm{e}^{t / T}}{(\beta+t)^{2}} \leq K \mathrm{e} .
$$

Now, set

$$
\bar{h}(t, m)= \begin{cases}K \mathrm{e}^{t / T+\beta m} & \text { for }(t, m) \in[0, T] \times \mathbb{R}_{-}, \\ 0 & \text { for }(t, m) \in[0, T] \times(0,+\infty) .\end{cases}
$$

Using assumption (9) and the fact that $J_{y}=0$ in $\mathbb{R}$ for all $y>0$, we observe that

$$
\int_{\mathbb{R}} J_{y}^{t}(m-y) \bar{h}(t, y) d y=0 \text { for any } t \in[0, T] \text { and } m \in(0,+\infty) .
$$

\footnotetext{
${ }^{1}$ With a slight abuse of notation, we also use this notation for functions which are not necessarily in $E$. Notice that the set $E$ is complete for the topology induced by \|\|$_{E}$.
} 
On the other hand, for any $(t, m) \in[0, T] \times \mathbb{R}_{-}$, it follows from (10) and (37) that

$$
\begin{aligned}
U\left(\int_{\mathbb{R}} J_{y}^{t}(m-y) \bar{h}(t, y) d y-\bar{h}(t, m)\right) & =U \bar{h}(t, m)\left(\int_{-\infty}^{0} J_{y}(m-y) \mathrm{e}^{(\beta+t)(y-m)} d y-1\right) \\
& \leq U \bar{h}(t, m) \int_{-\infty}^{0} \bar{J}(m-y) \mathrm{e}^{(\beta+t)(y-m)} d y \\
& \leq U \bar{h}(t, m) \int_{\mathbb{R}} \bar{J}(y) \mathrm{e}^{-(\beta+t) y} d y \\
& \leq U \bar{h}(t, m)\left(\int_{-\infty}^{0} \bar{J}(y) \mathrm{e}^{2 \beta|y|} d y+\|\bar{J}\|_{L^{1}(\mathbb{R})}\right) .
\end{aligned}
$$

Consider any real number $T$ such that

$$
0<T \leq \min \left\{\beta,\left(U \int_{-\infty}^{0} \bar{J}(y) \mathrm{e}^{2 \beta|y|} d y+U\|\bar{J}\|_{L^{1}(\mathbb{R})}+K \mathrm{e}\right)^{-1}\right\} .
$$

Therefore, using the inequalities of the previous paragraph together with (51), we get that, for all $(t, m) \in[0, T] \times \mathbb{R}$,

$$
\partial_{t} \bar{h}(t, m)=\frac{\bar{h}(t, m)}{T} \geq U\left(\int_{\mathbb{R}} J_{y}^{t}(m-y) \bar{h}(t, y) d y-\bar{h}(t, m)\right)+\left|\bar{m}_{v}(t)\right| \bar{h}(t, m) .
$$

Observe also that $\bar{h} \in \mathcal{C}^{1}\left([0, T], L_{-}^{\infty}(\mathbb{R})\right)$. In other words, $\bar{h} \in \mathcal{C}^{1}\left([0, T], L_{-}^{\infty}(\mathbb{R})\right)$ is a supersolution of problem (50) satisfied by $h=F(v)$. From the definition (46) of $K$ and from (7), we also know that $0 \leq h(0, m)=p_{0}(m) \leq \bar{h}(0, m)$ for a.e. $m \in \mathbb{R}$.

Using the comparison principle of Lemma 6.1, we obtain that, for every $t \in[0, T]$, $0 \leq h(t, m) \leq \bar{h}(t, m)$ for a.e. $m \in \mathbb{R}$. Together with Lemma 6.4, it follows that $h=F(v) \in E$.

Step 3: $F$ is a contraction mapping. Let $T>0$ be as in (53), let $v_{1}, v_{2} \in E$ and define $H=F\left(v_{1}\right)-F\left(v_{2}\right)$. Notice that the function $H$ belongs to $\mathcal{C}^{1}\left([0, T], L_{-}^{\infty}(\mathbb{R})\right)$ from Lemma 6.4 and that it satisfies, for all $t \in[0, T]$ and a.e. $m \in \mathbb{R}$,

$$
\left\{\begin{aligned}
\partial_{t} H(t, m) & =U\left(J_{y}^{t} \circledast H-H\right)(t, m)-\left(\bar{m}_{v_{1}}(t)-\bar{m}_{v_{2}}(t)\right) F\left(v_{1}\right)(t, m)-\bar{m}_{v_{2}}(t) H(t, m), \\
H(0, m) & =0 .
\end{aligned}\right.
$$

Define

$$
\bar{H}(t, m)= \begin{cases}\frac{\left\|v_{1}-v_{2}\right\|_{E}}{2} \mathrm{e}^{t / T+\beta m} & \text { for }(t, m) \in[0, T] \times \mathbb{R}_{-} \\ 0 & \text { for }(t, m) \in[0, T] \times(0,+\infty)\end{cases}
$$

Similar computations as for $\bar{h}$ and $v$ above imply that

$$
U\left(\int_{\mathbb{R}} J_{y}^{t}(m-y) \bar{H}(t, y) d y-\bar{H}(t, m)\right) \leq U \bar{H}(t, m)\left(\int_{-\infty}^{0} \bar{J}(y) \mathrm{e}^{2 \beta|y|} d y+\|\bar{J}\|_{L^{1}(\mathbb{R})}\right)
$$

for all $(t, m) \in[0, T] \times \mathbb{R}$, and that $\left|\bar{m}_{v_{1}}(t)-\bar{m}_{v_{2}}(t)\right| \leq \mathrm{e}\left\|v_{1}-v_{2}\right\|_{E}$ for all $t \in[0, T]$. Since $F\left(v_{1}\right) \in E$, it then follows that

$$
\left|\left(\bar{m}_{v_{1}}(t)-\bar{m}_{v_{2}}(t)\right) F\left(v_{1}\right)(t, m)\right| \leq K \mathrm{e}\left\|v_{1}-v_{2}\right\|_{E} \mathrm{e}^{t / T+\beta m}=2 K \mathrm{e} \bar{H}(t, m),
$$


for all $t \in[0, T]$ and a.e. $m \in \mathbb{R}_{-}$.

Finally, let us consider the real number $T$ defined by (47). Using (51), (55) and (56), we get that

$\partial_{t} \bar{H}(t, m)=\frac{\bar{H}(t, m)}{T} \geq U\left(J_{t} \circledast \bar{H}-\bar{H}\right)(t, m)+\left|\left(\bar{m}_{v_{1}}(t)-\bar{m}_{v_{2}}(t)\right) F\left(v_{1}\right)(t, m)\right|+\left|\bar{m}_{v_{2}}(t)\right| \bar{H}(t, m)$

for all $t \in[0, T]$ and $m \in \mathbb{R}_{-}$(and also for $m>0$ since all quantities are then equal to 0 ). As above, this implies that $\bar{H}$ is a supersolution of equation (54) satisfied by $H$, while $\bar{H} \in$ $\mathcal{C}^{1}\left([0, T], L_{-}^{\infty}(\mathbb{R})\right)$. The functions $h_{1}:=H$ and $h_{2}:=\bar{H}$ then satisfy some inequalities of the type (42) with $\varpi=-\bar{m}_{v_{2}}$ and $\tau:=T$. Using again the comparison principle of Lemma 6.1 , we get that

$$
H(t, \cdot) \leq \bar{H}(t, \cdot) \text { a.e. in } \mathbb{R} \text { for all } t \in[0, T] .
$$

Similarly, one has $-H(t, \cdot) \leq \bar{H}(t, \cdot)$ a.e. in $\mathbb{R}$ for all $t \in[0, T]$. This immediately yields

$$
\left\|F\left(v_{1}\right)-F\left(v_{2}\right)\right\|_{E} \leq \frac{1}{2}\left\|v_{1}-v_{2}\right\|_{E}
$$

Thus, $F: E \rightarrow E$ is a contraction mapping.

Step 4: conclusion. Consider now $T>0$ as defined in (47). The Banach fixed point theorem implies that $F$ admits a unique fixed point $v \in E$. This function $v$ then belongs to $\mathcal{C}^{1}\left([0, T], L_{-}^{\infty}(\mathbb{R})\right)$ from Lemma 6.4 , it satisfies

$$
0 \leq v(t, m) \leq K \mathrm{e}^{t / T+\beta m} \text { for all } t \in[0, T] \text { and a.e. } m \in \mathbb{R}
$$

with supp $v(t, \cdot) \subset \mathbb{R}_{-}$for every $t \in[0, T]$. This function $v$ is also the unique such solution of (48). Furthermore, with similar estimates as in (51) and (51), there is $K^{\prime}>0$ such that $\left|\partial_{t} v(t, m)\right| \leq K^{\prime} \mathrm{e}^{t / T+\beta m}$ for all $t \in[0, T]$ and a.e. $m \in \mathbb{R}_{-}$, and $\partial_{t} v(t, m)=0$ for all $t \in[0, T]$ and a.e. $m>0$.

Finally, letting

$$
p(t, m)=\mathrm{e}^{t m} v(t, m)
$$

for $(t, m) \in[0, T] \times \mathbb{R}$, it is straightforward to check that $p \in \mathcal{C}^{1}\left([0, T], L^{\infty}(\mathbb{R})\right.$ is a solution of $(3)$ in $[0, T] \times \mathbb{R}$, with $\operatorname{supp} p(t, \cdot) \subset \mathbb{R}_{-}$for every $t \in[0, T]$. Additionally, as $v \in E$, it follows that $p(t, \cdot) \in L^{1}(\mathbb{R})$ for all $t \in[0, T]$. Lastly, $p \in \mathcal{C}^{1}\left([0, T], L^{\infty}(\mathbb{R}) \cap L^{1}(\mathbb{R})\right)$, the function

$$
t \mapsto \bar{m}(t)=\int_{\mathbb{R}} m p(t, m) d m=\int_{-\infty}^{0} m p(t, m) d m
$$

is continuous in $[0, T]$ ), and

$$
0 \leq p(t, m) \leq K \mathrm{e}^{t / T+(\beta+t) m} \text { for all } t \in[0, T] \text { and a.e. } m \in \mathbb{R}_{-} .
$$

The proof of Proposition 6.2 is thereby complete.

Proof of Theorem 3.3. We are now in position to prove the global existence result of Theorem 3.3. Let $T_{\max }>0$ be the largest time such that equation (3) admits a solution 
$p \in \mathcal{C}^{1}\left(\left[0, T_{\max }\right), L^{\infty}(\mathbb{R}) \cap L^{1}(\mathbb{R})\right)$ with $\bar{m} \in \mathcal{C}\left(\left[0, T_{\max }\right)\right)$ and, for every $T \in\left(0, T_{\max }\right)$, there is $C_{T}>0$ such that

$$
0 \leq p(t, m) \leq C_{T} \mathrm{e}^{T m} \text { for all } t \in[0, T] \text { and a.e. } m \in \mathbb{R} .
$$

Notice that Proposition 3.1 then implies that $\operatorname{supp} p(t, \cdot) \subset \mathbb{R}_{-}$for every $t \in\left[0, T_{\max }\right)$. It follows from the local existence and uniqueness result of Proposition 6.2 applied with, say, $\beta=1$ that

$$
T_{\max } \in(0,+\infty]
$$

Notice that $T_{\max }$ does not depend on the choice $\beta=1$ in Proposition 6.2, since (57) does not involve any $\beta$. Our goal is to show that $T_{\max }=+\infty$.

We begin with some fundamental estimates stated in the following lemma, whose proof is postponed in Section 7 .

Lemma 6.5 (Mass preservation and estimates on the mean fitness) We have:

$$
\int_{\mathbb{R}} p(t, m) d m=\int_{-\infty}^{0} p(t, m) d m=1 \text { for all } t \in\left[0, T_{\max }\right)
$$

and

$$
\bar{m}(t) \geq \bar{m}(0)+t U \mu \text { for all } t \in\left[0, T_{\max }\right),
$$

with

$$
\mu=\int_{-\infty}^{0} m \bar{J}(m) d m \in(-\infty, 0)
$$

Assume now by contradiction that $T_{\max }<+\infty$. Define

$$
v(t, m)=p(t, m) \mathrm{e}^{-t m}
$$

for $(t, m) \in\left[0, T_{\max }\right) \times \mathbb{R}$. The function $v$ is a solution of the Cauchy problem (48) for all $T \in\left[0, T_{\max }\right)$ and, from (57) and the regularity properties of $p$, the function $v$ belongs to $\mathcal{C}^{1}\left(\left[0, T_{\max }\right), L_{-}^{\infty}(\mathbb{R})\right)$. Set now

$$
\beta_{0}=T_{\max }+1
$$

and let

$$
K_{0}=\underset{m \in \mathbb{R}}{\operatorname{ess} \sup }\left(p_{0}(m) \mathrm{e}^{-\beta_{0} m}\right)
$$

be defined as in (46) in Proposition 6.2 with the choice $\beta=\beta_{0}$. Denote

$$
\bar{v}(t, m)= \begin{cases}K_{0} \mathrm{e}^{\lambda t+\beta_{0} m} & \text { for }(t, m) \in\left[0, T_{\max }\right) \times \mathbb{R}_{-}, \\ 0 & \text { for }(t, m) \in\left[0, T_{\max }\right) \times(0,+\infty),\end{cases}
$$

where $\lambda \in \mathbb{R}$ is to be chosen later. Using (59) and the property $\bar{m}(t)=\bar{m}_{v}(t)$ for all $t \in$ $\left[0, T_{\max }\right.$ ), it is easily seen (as in Step 2 of the proof of Proposition 6.2) that, for every $T \in$ $\left(0, T_{\max }\right), \bar{v}$ is a supersolution of the equation (48) (for which $\bar{m}_{v}(t)=\bar{m}(t)$ is considered as a fixed coefficient) satisfied by $v$ on $[0, T]$, provided that

$$
\lambda \geq U \int_{-\infty}^{0} \bar{J}(m) \mathrm{e}^{2 \beta_{0}|m|} d m+U\|\bar{J}\|_{L^{1}(\mathbb{R})}-\bar{m}(0)-T U \mu .
$$


Let then

$$
\lambda^{\star}=U \int_{-\infty}^{0} \bar{J}(m) \mathrm{e}^{2 \beta_{0}|m|} d m+U\|\bar{J}\|_{L^{1}(\mathbb{R})}+|\bar{m}(0)|+T_{\text {max }} U|\mu|>0 .
$$

Using the comparison principle of Lemma 6.1 applied with every $\tau \in\left(0, T_{\max }\right)$, we obtain that

$$
\begin{cases}0 \leq v(t, m) \leq K_{0} \mathrm{e}^{\lambda^{\star} t+\beta_{0} m} & \text { for all } t \in\left[0, T_{\max }\right) \text { and a.e. } m \in \mathbb{R}_{-}, \\ \operatorname{supp} v(t, \cdot) \subset \mathbb{R}_{-} & \text {for all } t \in\left[0, T_{\max }\right) .\end{cases}
$$

Now, for any $\theta \in(0,1)$, set $p_{\theta}=p\left(\theta T_{\max }, \cdot\right)$. We have

$$
0 \leq p_{\theta}(m)=v\left(\theta T_{\max }, m\right) \mathrm{e}^{\theta T_{\max } m} \leq K_{0} \mathrm{e}^{\lambda^{\star} T_{\max }+\left(\theta T_{\max }+\beta_{0}\right) m}=K_{0} \mathrm{e}^{\lambda^{\star} T_{\max }+\left(\theta T_{\max }+T_{\max }+1\right) m}
$$

for a.e. $m \in \mathbb{R}_{-}$, while supp $p_{\theta} \subset \mathbb{R}_{-}$. For any $\theta \in(0,1)$, the function $p_{\theta}$ satisfies (5), owing to (57) and (58). Furthermore,

$$
0<K_{\theta}:=\operatorname{ess}_{m \in \mathbb{R}}\left(p_{\theta}(m) \mathrm{e}^{-\left(\theta T_{\max }+T_{\max }+1\right) m}\right) \leq K_{0} \mathrm{e}^{\lambda^{\star} T_{\max }}<+\infty .
$$

As a consequence, we can apply Proposition 6.2 with

$$
\beta=\theta T_{\max }+T_{\max }+1
$$

and the initial condition $p_{\theta}$. Thus, for any $\theta \in(0,1)$, there exist a time $T_{\theta}>0$, defined as in (47) with $K_{\theta}$ instead of $K$, and a unique solution $\tilde{p} \in \mathcal{C}^{1}\left(\left[0, T_{\theta}\right], L^{\infty}(\mathbb{R}) \cap L^{1}(\mathbb{R})\right)$ of (3) with initial condition $p_{\theta}$, such that

$$
0 \leq \tilde{p}(t, m) \leq K_{\theta} \mathrm{e}^{t / T_{\theta}+\left(\theta T_{\max }+T_{\max }+1+t\right) m}
$$

for all $t \in\left[0, T_{\theta}\right]$ and a.e. $m \in \mathbb{R}_{-}$, and $\operatorname{supp} \tilde{p}(t, \cdot) \subset \mathbb{R}_{-}$for every $t \in\left[0, T_{\theta}\right]$. Therefore, for any $\theta \in(0,1)$, problem (3) with initial condition $p_{0}$ has a solution $p \in \mathcal{C}^{1}\left(\left[0, \theta T_{\text {max }}+\right.\right.$ $\left.\left.T_{\theta}\right], L^{\infty}(\mathbb{R}) \cap L^{1}(\mathbb{R})\right)$ such that, for all $t \in\left[\theta T_{\max }, \theta T_{\max }+\min \left(T_{\theta}, 1\right)\right]$

$$
0 \leq p(t, m) \leq K_{\theta} \mathrm{e}^{\left(t-\theta T_{\max }\right) / T_{\theta}+\left(\theta T_{\max }+T_{\max }+1+t-\theta T_{\max }\right) m} \leq K_{0} \mathrm{e}^{\lambda^{\star} T_{\max }+1+\left(\theta T_{\max }+T_{\max }+1\right) m}
$$

for a.e. $m \in \mathbb{R}_{-}$, and $\operatorname{supp} p(t, \cdot) \subset \mathbb{R}_{-}$.

On the other hand, from (61) and from the definitions (62) of $\beta$ and (47) of $T_{\theta}>0$ with $K_{\theta}$ instead of $K$, it follows that

$$
\liminf _{\theta \rightarrow 1, \theta<1} T_{\theta}>0 .
$$

Therefore, there exist $\theta^{\prime} \in(0,1)$ and $T^{\prime} \in\left(T_{\max }, T_{\max }+1\right)$ for which problem (3) with initial condition $p_{0}$ has a solution $p \in \mathcal{C}^{1}\left(\left[0, T^{\prime}\right], L^{\infty}(\mathbb{R}) \cap L^{1}(\mathbb{R})\right)$ such that, for all $t \in\left[\theta^{\prime} T_{\text {max }}, T^{\prime}\right]$,

$$
0 \leq p(t, m) \leq K_{0} \mathrm{e}^{\lambda^{\star} T_{\max }+1+\left(\theta^{\prime} T_{\max }+T_{\max }+1\right) m} \leq K_{0} \mathrm{e}^{\lambda^{\star} T_{\max }+1+T^{\prime} m}
$$

for a.e. $m \in \mathbb{R}_{-}$, together with $\operatorname{supp} p(t, \cdot) \subset \mathbb{R}_{-}$. Furthermore, (60) (remember that $\beta_{0}=$ $\left.T_{\text {max }}+1\right)$ implies that, for all $t \in\left[0, \theta^{\prime} T_{\max }\right]$ and a.e. $m \in \mathbb{R}_{-}$,

$$
0 \leq p(t, m)=v(t, m) e^{t m} \leq K_{0} \mathrm{e}^{\lambda^{\star} \theta^{\prime} T_{\max }+\left(T_{\max }+1\right) m} \leq K_{0} \mathrm{e}^{\lambda^{\star} \theta^{\prime} T_{\max }+T^{\prime} m},
$$

and $\operatorname{supp} p(t, \cdot) \subset \mathbb{R}_{-}$for all $t \in\left[0, \theta^{\prime} T_{\max }\right]$. Together with (63) in $\left[\theta^{\prime} T_{\text {max }}, T^{\prime}\right] \times \mathbb{R}_{-}$, it follows that the solution $p$ satisfies (57) for all $T \in\left(0, T^{\prime}\right]$. Finally, one infers that $\bar{m} \in \mathcal{C}\left(\left[0, T^{\prime}\right)\right)$. The fact that $T^{\prime}$ is larger than $T_{\max }$ contradicts the definition of $T_{\max }$.

As a conclusion, $T_{\max }=+\infty$ and, from (57) holding for any $T>0$, property (20) holds with $\Gamma_{\alpha, T}=C_{\max (\alpha, T)}$. From the equation (3) itself and from (10), it also follows that $\left|\partial_{t} p(t, m)\right|$ decays faster than any exponential function as $m \rightarrow-\infty$ in the sense that (20) holds for $\left|\partial_{t} p(t, m)\right|$ as well. The proof of Theorem 3.3 is thereby complete. 


\subsection{Proof of the results on the stationary states of (24) and (33)}

Proof of Proposition 3.5. Let us first show that $\sup \left\{\operatorname{supp} p_{\infty}\right\}=0$. Assume not. Then there is $\delta>0$ such that

$$
p_{\infty}((-\delta, 0])=0 \text { and } p_{\infty}([-\delta-\varepsilon,-\delta])>0 \text { for all } \varepsilon>0 .
$$

Consider now any nonnegative continuous function $\phi: \mathbb{R} \rightarrow \mathbb{R}$ with compact support included in $[-\delta, 0]$, and such that

$$
\int_{-\delta}^{-\delta+\eta} \phi(m) d m>0 \text { for all } \eta>0
$$

Then, using (10) with $\bar{J} \in L^{1}(\mathbb{R})$, and the fact that the function $y \mapsto \int_{\mathbb{R}} J_{y}(m-y) \phi(m) d m$ is continuous (and bounded), it follows (as in the derivation of (33)) that

$$
\begin{aligned}
0= & \int_{\mathbb{R}} \int_{\mathbb{R}} J_{y}(m-y) \phi(m) d m d p_{\infty}(y)-U \int_{\mathbb{R}} \phi(m) d p_{\infty}(m) \\
& +\int_{\mathbb{R}} m \phi(m) d p_{\infty}(m)-\bar{m}_{\infty} \int_{\mathbb{R}} \phi(m) d p_{\infty}(m) .
\end{aligned}
$$

Since the measure $p_{\infty}$ is supported in $(-\infty,-\delta]$ and the continuous function $\phi$ is supported in $[-\delta, 0]$, one infers from the previous formula that

$$
\int_{\mathbb{R}} \int_{\mathbb{R}} J_{y}(m-y) \phi(m) d m d p_{\infty}(y)=0 .
$$

From the assumption (18) on the positivity and continuity of $y \mapsto S_{y}$ on $(-\infty, 0)$, there is $\varepsilon \in(0, \delta)$ such that $2 \varepsilon<S_{y}$ for all $y \in[-\delta-\varepsilon,-\delta]$. It then follows from (66) and the nonnegativity of $J_{y}, \phi$ and $p_{\infty}$ that

$$
\int_{-\delta-\varepsilon}^{-\delta} \int_{-\delta}^{-\delta+\varepsilon} J_{y}(m-y) \phi(m) d m d p_{\infty}(y)=0 .
$$

On the other hand, for each $y \in[-\delta-\varepsilon,-\delta]$ and each $m \in[-\delta,-\delta+\varepsilon]$, one has $0 \leq m-y \leq$ $2 \varepsilon<S_{y}$. Hence, for every $y \in[-\delta-\varepsilon,-\delta]$, one has $J_{y}(\cdot-y)>0$ a.e. in $[-\delta,-\delta+\varepsilon]$. Together with (65) and the nonnegativity of $\phi$, it follows that

$$
\int_{-\delta}^{-\delta+\varepsilon} J_{y}(m-y) \phi(m) d m>0 \text { for all } y \in[-\delta-\varepsilon,-\delta] .
$$

Together with (67) and the nonnegativity of $p_{\infty}$, one gets that $p_{\infty}([-\delta-\varepsilon,-\delta])=0$, a contradiction with (64). Therefore, one has shown that

$$
\sup \left\{\operatorname{supp} p_{\infty}\right\}=0 \text {. }
$$

Lemma 4.5 in $[17]^{2}$ then implies that the CGF $C_{\infty}$ of $p_{\infty}$ satisfies $C_{\infty}^{\prime}(z) \rightarrow 0$ as $z \rightarrow+\infty$.

Finally, let us prove that $\bar{m}_{\infty} \geq-U$. Using equation (33) satisfied by $C_{\infty}$ and the fact that $\bar{m}_{\infty}=C_{\infty}^{\prime}(0)$, we obtain that

$$
\bar{m}_{\infty}+U=C_{\infty}^{\prime}(0)+U=C_{\infty}^{\prime}(z)+U \mathrm{e}^{C_{\infty}(z+\omega(z))-C_{\infty}(z)} M_{\star}(z) \geq C_{\infty}^{\prime}(z)
$$

\footnotetext{
${ }^{2}$ It is immediate to see that the proof and the conclusion of [17, Lemma 4.5] hold good even if the nonnegative probability measure $p_{\infty}$ is not in $L^{\infty}(\mathbb{R})$.
} 
for all $z \geq 0$. Thus, by passing to the limit as $z \rightarrow+\infty$, one immediately gets that $\bar{m}_{\infty}+U \geq 0$. The proof of Proposition 3.5 is thereby complete.

Proof of Proposition 3.6. Equation (33) satisfied by $C_{\infty}$ can be rewritten as

$$
\mathrm{e}^{C_{\infty}(z)}\left(C_{\infty}^{\prime}(0)+U-C_{\infty}^{\prime}(z)\right)=U \mathrm{e}^{C_{\infty}(z+\omega(z))} M_{\star}(z)
$$

for all $z \geq 0$. On the one hand we have

$$
\lim _{z \rightarrow+\infty} \mathrm{e}^{C_{\infty}(z)}=\lim _{z \rightarrow+\infty} \int_{-\infty}^{0} \mathrm{e}^{z m} d p_{\infty}(m)=\rho
$$

from the definition of $\rho \in[0,1]$ in (27). On the other hand, we know from the previous proposition that $C_{\infty}^{\prime}(z) \rightarrow 0$ as $z \rightarrow+\infty$. By passing the limit as $z \rightarrow+\infty$ in (68), we get that the right-hand side has a limit and that

$$
\rho\left(C_{\infty}^{\prime}(0)+U\right)=U \lim _{z \rightarrow+\infty} \mathrm{e}^{C_{\infty}(z+\omega(z))} M_{\star}(z)
$$

Since

$$
M_{\star}(z)=\int_{-\infty}^{0} J_{0}(m) \mathrm{e}^{m z} d m
$$

is the MGF of the mutation kernel $J_{0}$ at the optimal fitness, with $J_{0} \in L^{1}(\mathbb{R})$, one has $\lim _{z \rightarrow+\infty} M_{\star}(z)=0$. Moreover, the function $s \mapsto \mathrm{e}^{C_{\infty}(s)}$ is continuous in $[0,+\infty)$ and converges to $\rho \in[0,1]$ at $+\infty$. Thus, it is bounded in $[0,+\infty)$. Therefore, we get that $\lim _{z \rightarrow+\infty} \mathrm{e}^{C_{\infty}(z+\omega(z))} M_{\star}(z)=0$ and

$$
\rho\left(\bar{m}_{\infty}+U\right)=\rho\left(C_{\infty}^{\prime}(0)+U\right)=0
$$

that is to say that $\rho=0$ or $\bar{m}_{\infty}=-U$.

Proof of Proposition 3.7. Let us consider the MGF of $p_{\infty}$, namely the function defined in $\mathbb{R}_{+}$by

$$
M_{\infty}(z)=\mathrm{e}^{C_{\infty}(z)}=\int_{\mathbb{R}} \mathrm{e}^{z m} d p_{\infty}(m)=\int_{-\infty}^{0} \mathrm{e}^{z m} d p_{\infty}(m), \text { for } z \geq 0 .
$$

Equations (33) and (68) with $C_{\infty}^{\prime}(0)=\bar{m}_{\infty}$ can be rewritten as

$$
M_{\infty}(z)\left(\bar{m}_{\infty}+U\right)-M_{\infty}^{\prime}(z)=U \mathrm{e}^{C_{\infty}(z+\omega(z))} M_{\star}(z) .
$$

We then consider separately the cases $s_{H}^{\star}=0$ and $s_{H}^{\star} \neq 0$, where $s_{H}^{\star}$ is defined in (28).

First case: $s_{H}^{\star}=0$. This case means that

$$
\int_{0}^{+\infty} M_{\star}(z) d z=+\infty .
$$

Meanwhile, due to (8), (9) and (11), the function $\omega$ necessarily satisfies some properties, whose proof is postponed in Appendix B below:

$$
\omega(0)=0, \omega(z) \geq-z \text { and } \omega^{\prime \prime}(z) \leq 0 \text { for all } z \geq 0 .
$$


Thus, it follows the function $z \mapsto \omega(z)+z$ is nonnegative and nondecreasing. It then has a limit $l \in[0,+\infty]$ as $z \rightarrow+\infty$. Assume first here that

$$
\lim _{z \rightarrow+\infty} z+\omega(z)=l \in[0,+\infty) .
$$

Thus, $\mathrm{e}^{C_{\infty}(z+\omega(z))} M_{\star}(z) \sim \mathrm{e}^{C_{\infty}(l)} M_{\star}(z)$ as $z \rightarrow+\infty$ and

$$
\int_{0}^{+\infty} \mathrm{e}^{C_{\infty}(z+\omega(z))} M_{\star}(z) d z=+\infty .
$$

Since $\int_{0}^{+\infty} M_{\infty}^{\prime}(z) d z=\rho-1$ and $M_{\infty}(z)>0$ for all $z \in[0,+\infty)$, it follows from (69) and (72) that $\bar{m}_{\infty}+U>0$. From Proposition 3.6, this means that $\rho=0$.

Assume now that

$$
\lim _{z \rightarrow+\infty} z+\omega(z)=+\infty
$$

Since $C_{\infty}$ is nonincreasing in $[0,+\infty)$ from its definition (32) and since $\omega$ is nonpositive in $[0,+\infty)$ by $(12)$ and $(71)$, we get that $\mathrm{e}^{C_{\infty}(z+\omega(z))-C_{\infty}(z)} \geq 1$ for all $z \geq 0$. Thus, using (70), one infers that

$$
\int_{0}^{+\infty} \mathrm{e}^{C_{\infty}(z+\omega(z))-C_{\infty}(z)} M_{\star}(z) d z \geq \int_{0}^{+\infty} M_{\star}(z) d z=+\infty .
$$

Let us then show that $\rho=0$. If $\bar{m}_{\infty}+U>0$, then Proposition 3.6 yields $\rho=0$. Assume now that $\bar{m}_{\infty}+U=0$, in other words $C_{\infty}^{\prime}(0)+U=0$. By integrating (33) over $(0, A)$ with $A>0$, we obtain that

$$
\int_{0}^{A} \mathrm{e}^{C_{\infty}(z+\omega(z))-C_{\infty}(z)} M_{\star}(z) d z=C_{\infty}(0)-C_{\infty}(A)=-C_{\infty}(A),
$$

that is,

$$
M_{\infty}(A)=\mathrm{e}^{C_{\infty}(A)}=\exp \left(-\int_{0}^{A} \mathrm{e}^{C_{\infty}(z+\omega(z))-C_{\infty}(z)} M_{\star}(z) d z\right) .
$$

Knowing that $\lim _{A \rightarrow+\infty} M_{\infty}(A)=\rho$ and using (73), we finally get that

$$
\rho=\lim _{A \rightarrow+\infty} \exp \left(-\int_{0}^{A} \mathrm{e}^{C_{\infty}(z+\omega(z))-C_{\infty}(z)} M_{\star}(z) d z\right)=0 .
$$

Second case: $s_{H}^{\star} \neq 0$. This case means that

$$
0<\int_{0}^{+\infty} M_{\star}(z) d z=\frac{1}{s_{H}^{\star}}<+\infty .
$$

Since $C_{\infty}$ is nonpositive by $(32)$ and $p_{\infty}((-\infty, 0])=1$, we also have that

$$
0<\int_{0}^{+\infty} M_{\star}(z) \mathrm{e}^{C_{\infty}(z+\omega(z))} d z \leq \int_{0}^{+\infty} M_{\star}(z) d z=\frac{1}{s_{H}^{\star}}<+\infty .
$$

Using the inequality $\bar{m}_{\infty}+U \geq 0$ and integrating $(69)$ over $(0,+\infty)$ yields

$$
0 \leq\left(\bar{m}_{\infty}+U\right) \int_{0}^{+\infty} M_{\infty}(z) d z=\rho-1+U \int_{0}^{+\infty} M_{\star}(z) \mathrm{e}^{C_{\infty}(z+\omega(z))} d z \leq \rho-1+\frac{U}{s_{H}^{\star}} .
$$


Therefore, if $U<s_{H}^{\star}$, then $\rho>0$, and $\bar{m}_{\infty}=-U$ by Proposition 3.6.

Let us now consider the set

$$
E=\left\{U \geq s_{H}^{\star}: \text { there is } z>0 \text { with } 1+U \omega(z) M_{\star}(z)=0\right\} .
$$

Remember that $M_{\star}(z)>0$ for all $z \geq 0$. Furthermore, as $\omega$ satisfies (12) and (71), there is $z_{0}>0$ such that $\omega<0$ in $\left[z_{0},+\infty\right)$. Therefore, for every $U \geq s_{H}^{\star}$ such that $U>-1 /\left(\omega\left(z_{0}\right) M_{\star}\left(z_{0}\right)\right)(>0)$, there holds $1+U \omega\left(z_{0}\right) M_{\star}\left(z_{0}\right)<0$ and, by continuity of $\omega$ and $M_{\star}$ and since $\omega(0)=0$, there is $z_{1}>0$ such that $1+U \omega\left(z_{1}\right) M_{\star}\left(z_{1}\right)=0$. As a consequence, the set $E$ is not empty and

$$
U_{c}:=\inf E \in\left[s_{H}^{\star},+\infty\right) .
$$

Pick any $U>U_{c}$. We want to show that $\rho=0$ and $\bar{m}_{\infty}>-U$. Assume by contradiction that $\bar{m}_{\infty}=-U$, that is, $C_{\infty}^{\prime}(0)=-U$. Using (33), we obtain that, for all $z \geq 0$,

$$
-C_{\infty}^{\prime}(z)=U \mathrm{e}^{C_{\infty}(z+\omega(z))-C_{\infty}(z)} M_{\star}(z) \geq U\left(1+. C_{\infty}(z+\omega(z))-C_{\infty}(z)\right) M_{\star}(z) .
$$

Using (71) and the convexity of $C_{\infty}$ in $[0,+\infty)$, one infers that, for all $z \geq 0, C_{\infty}(z+\omega(z))-$ $C_{\infty}(z) \geq C_{\infty}^{\prime}(z) \omega(z)$, hence $-C_{\infty}^{\prime}(z) \geq U\left(1+C_{\infty}^{\prime}(z) \omega(z)\right) M_{\star}(z)$, that is,

$$
-C_{\infty}^{\prime}(z)\left(1+U \omega(z) M_{\star}(z)\right)-U M_{\star}(z) \geq 0 .
$$

On the other hand, as $U>U_{c}$, there are $U^{\prime} \in\left[s_{H}^{\star}, U\right) \subset(0, U)$ and $z^{\prime}>0$ such that $1+U^{\prime} \omega\left(z^{\prime}\right) M_{\star}\left(z^{\prime}\right)=0$, hence $\omega\left(z^{\prime}\right) M_{\star}\left(z^{\prime}\right)=-1 / U^{\prime}<0$ and $1+U \omega\left(z^{\prime}\right) M_{\star}\left(z^{\prime}\right)<0$. Again by continuity, one infers the existence of $z_{1}>0$ such that

$$
1+U \omega\left(z_{1}\right) M_{\star}\left(z_{1}\right)=0 .
$$

Formula (76) at $z=z_{1}$ yields $-U M_{\star}\left(z_{1}\right) \geq 0$, which is ruled out since $U>0$ and $M_{\star}\left(z_{1}\right)>0$. Thus, $\bar{m}_{\infty}>-U$, and $\rho=0$ by Proposition 3.6. The proof of Proposition 3.7 is thereby complete.

Proof of Corollary 3.8. Using (31), it is easily seen that $s_{H}^{\star}=0$ is equivalent to $n \leq 2$. Furthermore, in the Gaussian Fisher's geometric model, $\omega(z)=-\lambda z^{2} /(1+\lambda z)$. Thus,

$$
\lim _{z \rightarrow+\infty} z+\omega(z)=\frac{1}{\lambda}<+\infty
$$

Together with Proposition 3.7, we get the conclusion (i) of Corollary 3.8 in case $n \leq 2$.

In case $n>2$, then $s_{H}^{\star}=\lambda(n-2) / 2>0$. Using again Proposition 3.7, we immediately get that $\rho>0$ and $\bar{m}_{\infty}=-U$ if $U<\lambda(n-2) / 2$.

Let us show that the same result holds good with the large inequality $U \leq \lambda(n-2) / 2=s_{H}^{\star}$ (still with $n>2$ ). First of all, by using Proposition 3.6 and equation (69) multiplied by $\rho$, one has

$$
-\rho M_{\infty}^{\prime}(z)=\rho U \mathrm{e}^{C_{\infty}(z+\omega(z))} M_{\star}(z)
$$

for all $z \geq 0$. Hence, afther integration over $(0,+\infty)$, one infers that

$$
\rho(1-\rho)=\rho U \int_{0}^{+\infty} \mathrm{e}^{C_{\infty}(z+\omega(z))} M_{\star}(z) d z .
$$


Therefore, $\rho<1$. As a consequence, the function $C_{\infty}$ defined in (32) (with $p_{\infty}$ written as in $(27))$ is (strictly) decreasing in $[0,+\infty)$ and thus negative in $(0,+\infty)$. Notice that these properties hold under the general assumptions of Proposition 3.7. On the other hand, since, here in the Gaussian Fisher's geometric model, $\omega(z)=-\lambda z^{2} /(1+\lambda z)$ for all $z \geq 0$, one has $z+\omega(z)>0$ for all $z>0$, hence $\mathrm{e}^{C_{\infty}(z+\omega(z))}<1$ for all $z>0$. Together with the positivity of $M_{\star}(z)$, it follows that

$$
0<\int_{0}^{+\infty} M_{\star}(z) \mathrm{e}^{C_{\infty}(z+\omega(z))} d z<\int_{0}^{+\infty} M_{\star}(z) d z=\frac{1}{s_{H}^{\star}}<+\infty .
$$

As a consequence, if $U \leq \lambda(n-2) / 2=s_{H}^{\star}$, then, as in (74), one gets that

$$
0 \leq\left(\bar{m}_{\infty}+U\right) \int_{0}^{+\infty} M_{\infty}(z) d z=\rho-1+U \int_{0}^{+\infty} M_{\star}(z) \mathrm{e}^{C_{\infty}(z+\omega(z))} d z<\rho-1+\frac{U}{s_{H}^{\star}} \leq \rho,
$$

hence $\rho>0$, and $\bar{m}_{\infty}=-U$ by Proposition 3.6.

Lastly, still in case $n>2$, a straightforward computation, using (13), implies that the quantity $U_{c}$ defined in $(75)$ is equal to

$$
U_{c}=\frac{\lambda(n+2)^{n / 2+1}}{16(n-2)^{n / 2-1}}
$$

Conclusion (ii)-(b) of Corollary 3.8 then follows immediately from Proposition 3.7.

\section{$7 \quad$ Proof of technical lemmas}

This section is devoted to the proof of the technical lemmas, namely Lemmas 6.1, 6.4 and 6.5, used in the proof of the main theorems in the previous sections.

\subsection{Comparison principle}

Proof of Lemma 6.1. Let $\tau, \varpi, h_{1}$ and $h_{2}$ satisfy the assumptions of the lemma. With a slight abuse of notations, we write $h_{1}(t)=h_{1}(t, \cdot) \in L_{-}^{\infty}(\mathbb{R})$ and $h_{2}(t)=h_{2}(t, \cdot) \in L_{-}^{\infty}(\mathbb{R})$ for $t \in[0, \tau]$. Set

$$
\mathcal{L}_{t}(h):=U\left(J_{y}^{t} \circledast h-h\right)-\varpi(t) h
$$

for $t \in[0, \tau]$ and $h \in L_{-}^{\infty}(\mathbb{R})$. For any such $h \in L_{-}^{\infty}(\mathbb{R})$, one has supp $\mathcal{L}_{t}(h) \subset \mathbb{R}_{-}$and, from (8), (10), (37) and similar calculations as in (52), there holds

$$
\left\|J_{y}^{t} \circledast h\right\|_{L^{\infty}(\mathbb{R})} \leq\|h\|_{L^{\infty}(\mathbb{R})}\left(\int_{-\infty}^{0} \bar{J}(y) \mathrm{e}^{t|y|} d y+\|\bar{J}\|_{L^{1}(\mathbb{R})}\right),
$$

hence, $\mathcal{L}_{t}(h) \in L_{-}^{\infty}(\mathbb{R})$. Then let us denote

$$
a(t)=\left(h_{2}^{\prime}(t)-\mathcal{L}_{t}\left(h_{2}(t)\right)\right)-\left(h_{1}^{\prime}(t)-\mathcal{L}_{t}\left(h_{1}(t)\right)\right)
$$

for $t \in[0, \tau]$. Notice that $\operatorname{supp} a(t) \subset \mathbb{R}_{-}$for every $t \in[0, \tau]$. Furthermore, for any $i \in\{1,2\}$, any $t \in[0, \tau]$ any sequence $\left(t_{n}\right)_{n \in \mathbb{N}}$ in $[0, \tau]$ converging to $t$, any $m \in \mathbb{R}_{-}$and any $n \in \mathbb{N}$, one 
has

$$
\begin{aligned}
& \left|\left(J_{y}^{t_{n}} \circledast h_{i}\right)\left(t_{n}, m\right)-\left(J_{y}^{t} \circledast h_{i}\right)(t, m)\right| \leq \int_{\mathbb{R}} J_{y}(m-y) \mathrm{e}^{-t_{n}(m-y)}\left|h_{i}\left(t_{n}, y\right)-h_{i}(t, y)\right| d y \\
& +\int_{\mathbb{R}} J_{y}(m-y)\left|\mathrm{e}^{-t_{n}(m-y)}-\mathrm{e}^{-t(m-y)}\right|\left|h_{i}(t, y)\right| d y \\
& \leq\left\|h_{i}\left(t_{n}, \cdot\right)-h_{i}(t, \cdot)\right\|_{L^{\infty}(\mathbb{R})}\left(\int_{-\infty}^{0} \bar{J}(y) \mathrm{e}^{\tau|y|} d y+\|\bar{J}\|_{L^{1}(\mathbb{R})}\right) \\
& +\left\|h_{i}(t, \cdot)\right\|_{L^{\infty}(\mathbb{R})} \int_{\mathbb{R}} \bar{J}(y)\left|\mathrm{e}^{-t_{n} y}-\mathrm{e}^{-t y}\right| d y .
\end{aligned}
$$

Therefore, $\left\|J_{y}^{t_{n}} \circledast h_{i}\left(t_{n}\right)-J_{y}^{t} \circledast h_{i}(t)\right\|_{L^{\infty}(\mathbb{R})} \rightarrow 0$ as $n \rightarrow+\infty$ from the assumptions on $h_{i}$ and $\bar{J}$ and from Lebesgue's dominated convergence theorem. Finally, one infers that the maps $t \mapsto \mathcal{L}_{t}\left(h_{i}(t)\right)$ belong to $\mathcal{C}\left([0, \tau], L_{-}^{\infty}(\mathbb{R})\right)$, and that $a \in \mathcal{C}\left([0, \tau], L_{-}^{\infty}(\mathbb{R})\right)$.

Now define, for $t \in[0, \tau]$,

$$
w(t)=\left(h_{2}(t)-h_{1}(t)\right) \mathrm{e}^{K t} \in L_{-}^{\infty}(\mathbb{R}),
$$

with

$$
K=U+\max _{[0, \tau]}|\varpi| .
$$

It is straightforward to check that $w$ is a solution of the ordinary differential equation

$$
w^{\prime}(t)=F(t, w), \quad t \in[0, \tau],
$$

in $L_{-}^{\infty}(\mathbb{R})$, for some function $F:[0, \tau] \times L_{-}^{\infty}(\mathbb{R}) \rightarrow L_{-}^{\infty}(\mathbb{R})$ defined by

$$
F(t, w)=U J_{y}^{t} \circledast w+w(K-U-\varpi(t))+a(t) \mathrm{e}^{K t} .
$$

As above, the function $F$ is continuous in $[0, \tau] \times L_{-}^{\infty}(\mathbb{R})$. Furthermore, for any $t \in[0, \tau]$ and $w, w^{\prime} \in L_{-}^{\infty}(\mathbb{R})$, one has, as in $(77)$,

$$
\begin{aligned}
\left\|J_{y}^{t} \circledast w-J_{y}^{t} \circledast w^{\prime}\right\|_{L^{\infty}(\mathbb{R})} & \leq\left\|w-w^{\prime}\right\|_{L^{\infty}(\mathbb{R})}\left(\int_{-\infty}^{0} \bar{J}(y) \mathrm{e}^{t|y|} d y+\|\bar{J}\|_{L^{1}(\mathbb{R})}\right) \\
& \leq\left\|w-w^{\prime}\right\|_{L^{\infty}(\mathbb{R})}\left(\int_{-\infty}^{0} \bar{J}(y) \mathrm{e}^{\tau|y|} d y+\|\bar{J}\|_{L^{1}(\mathbb{R})}\right) .
\end{aligned}
$$

As a consequence, the function $F$ is Lipschitz-continuous with respect to $w$ uniformly in $t \in[0, \tau]$. We can then define $\widetilde{w} \in \mathcal{C}^{1}\left([0, \tau], L_{-}^{\infty}(\mathbb{R})\right)$ as the unique solution of $\widetilde{w}^{\prime}(t)=$ $\max \{F(t, \widetilde{w}(t)), 0\}$ in $[0, \tau]$ with $\widetilde{w}(0)=w(0)$, that is,

$$
\widetilde{w}(t)=w(0)+\int_{0}^{t} \max \{F(s, \widetilde{w}(s)), 0\} d s .
$$

We have $\widetilde{w}(t) \geq w(0)$ and $w(0) \geq 0$ a.e. in $\mathbb{R}$ by assumption. Additionally, from (42), there holds $a(t) \geq 0$ a.e. in $\mathbb{R}_{-}$, for all $t \in[0, \tau]$. As a consequence, and since $K-U-\varpi(t) \geq 0$ for all $[0, \tau]$, one infers that, for all $t \in[0, \tau], F(t, \widetilde{w}(t)) \geq 0$ a.e. in $\mathbb{R}_{-}$. We deduce that $\widetilde{w}$ is also a solution of the equation (78) satisfied by $w$. From the Cauchy-Lipschitz theorem, we deduce that, for all $t \in[0, \tau], w(t)=\widetilde{w}(t) \geq 0$ and therefore $h_{1}(t, \cdot) \leq h_{2}(t, \cdot)$ a.e. in $\mathbb{R}$. The proof of Lemma 6.1 is thereby complete. 


\subsection{Existence for the Cauchy problem}

Proof of Lemma 6.4. Let $v \in E$. We know by definition of the set $E$ and Lebesgue's dominated convergence theorem that the function

$$
t \mapsto \bar{m}_{v}(t)=\int_{-\infty}^{0} m v(t, m) \mathrm{e}^{t m} d m
$$

is continuous in $[0, T]$. Problem (50) can then be written as an ordinary differential equation

$$
\left\{\begin{array}{l}
h^{\prime}(t)=G(t, h), \quad t \in[0, T], \\
h(0)=p_{0}
\end{array}\right.
$$

with

$$
\begin{aligned}
G:[0, T] \times L_{-}^{\infty}(\mathbb{R}) & \rightarrow L_{-}^{\infty}(\mathbb{R}) \\
(t, X) & \mapsto U\left(J_{y}^{t} \circledast X-X\right)-\bar{m}_{v}(t) X .
\end{aligned}
$$

The function space $L_{-}^{\infty}\left(\mathbb{R}_{-}\right)$is a Banach space for the uniform norm \|\|$_{\infty}$ and as in the proof of Lemma 6.1 above, the function $G$ is continuous in $[0, T] \times L_{-}^{\infty}(\mathbb{R})$ and Lipschitz continuous with respect to $X$ uniformly in $t \in[0, \tau]$. Therefore, the Cauchy-Lipschitz theorem yields the existence and uniqueness of a solution $h \in \mathcal{C}^{1}\left([0, T], L_{-}^{\infty}(\mathbb{R})\right)$ of problem (79).

\subsection{Mass preservation and estimates on mean fitness}

Proof of Lemma 6.5. Let us first show (58). We consider

$$
\phi(t)=\int_{\mathbb{R}} p(t, m) d m=\int_{-\infty}^{0} p(t, m) d m
$$

for $t \in\left[0, T_{\max }\right)$. This quantity is a well-defined nonnegative real number due to (57) and the definition of $T_{\max }$. By using (57) for every $T \in\left[0, T_{\max }\right)$, integrating (3) against $m$ over $\mathbb{R}_{-}$, we obtain that $\phi$ is of class $\mathcal{C}^{1}\left(\left[0, T_{\max }\right)\right)$ and, for every $t \in\left[0, T_{\max }\right)$,

$$
\phi^{\prime}(t)=\int_{-\infty}^{0} \partial_{t} p(t, m) d m=U \int_{-\infty}^{0}\left(J_{y} \circledast p\right)(t, m) d m-U \phi(t)+\bar{m}(t)-\bar{m}(t) \phi(t) .
$$

From assumption (8), we have

$$
\int_{-\infty}^{0}\left(J_{y} \circledast p\right)(t, m) d m=\int_{-\infty}^{0}\left(\int_{-\infty}^{0} J_{y}(m-y) d m\right) p(t, y) d y=\int_{-\infty}^{0} p(t, y) d y=\phi(t) .
$$

Finally, $\phi^{\prime}(t)=\bar{m}(t)(1-\phi(t))$ for all $t \in\left[0, T_{\max }\right)$. From assumption (5), there holds $\phi(0)=1$, and since $\bar{m} \in \mathcal{C}\left(\left[0, T_{\max }\right)\right)$, it follows immediately that $\phi(t)=1$ for all $t \in\left[0, T_{\max }\right)$.

Let us now turn to the proof of (59). By integrating (3) between 0 and $t \in\left[0, T_{\max }\right)$, multiplying by $m$, integrating over $\mathbb{R}_{-}$and using (57) and Fubini's theorem, we get that, for every $t \in\left[0, T_{\max }\right)$,

$$
\begin{aligned}
\bar{m}(t)-\bar{m}(0) & =\int_{-\infty}^{0} \int_{0}^{t} m\left[U\left(\left(J_{y} \circledast p\right)(s, m)-p(s, m)\right)+(m-\bar{m}(s)) p(s, m)\right] d s d m \\
& =\int_{0}^{t} \int_{-\infty}^{0} m\left[U\left(\left(J_{y} \circledast p\right)(s, m)-p(s, m)\right)+(m-\bar{m}(s)) p(s, m)\right] d m d s .
\end{aligned}
$$


From $(8),(10),(57),(58)$ and Fubini's theorem, one infers that, for every $s \in[0, t]\left(\subset\left[0, T_{\max }\right)\right)$,

$$
\begin{aligned}
\int_{-\infty}^{0} m\left(J_{y} \circledast p\right)(s, m) d m= & \int_{-\infty}^{0}\left(\int_{-\infty}^{0}(m-y+y) J_{y}(m-y) p(s, y) d y\right) d m \\
= & \int_{-\infty}^{0}\left(\int_{-\infty}^{-y} m J_{y}(m) d m\right) p(s, y) d y \\
& +\int_{-\infty}^{0} y p(s, y)\left(\int_{-\infty}^{0} J_{y}(m-y) d m\right) d y \\
= & \int_{-\infty}^{0} \mu_{J_{y}} p(s, y) d y+\bar{m}(s)
\end{aligned}
$$

with

$$
\mu_{J_{y}}=\int_{-\infty}^{-y} m J_{y}(m) d m
$$

Then, using the fact that the functions $\bar{m}: t \mapsto \int_{-\infty}^{0} m p(t, m) d m$ and $t \mapsto \int_{\infty}^{0} m^{2} p(t, m) d m$ are continuous in $\left[0, T_{\max }\right)$, we deduce that, for every $t \in\left[0, T_{\max }\right)$,

$$
\bar{m}(t)-\bar{m}(0)=U \int_{0}^{t} \int_{-\infty}^{0} \mu_{J_{y}} p(s, y) d y d s+\int_{0}^{t} \int_{-\infty}^{0} m^{2} p(s, m) d m d s-\int_{0}^{t}(\bar{m}(s))^{2} d s .
$$

Then, by the Cauchy-Schwarz inequality and (58), we have

$$
\begin{aligned}
(\bar{m}(s))^{2} & =\left(\int_{-\infty}^{0} m p(s, m) d m\right)^{2} \\
& \leq\left(\int_{-\infty}^{0} p(s, m) d m\right)\left(\int_{-\infty}^{0} m^{2} p(s, m) d m\right)=\int_{-\infty}^{0} m^{2} p(s, m) d m
\end{aligned}
$$

for all $s \in[0, t]\left(\subset\left[0, T_{\max }\right)\right)$. Therefore,

$$
\bar{m}(t) \geq \bar{m}(0)+U \int_{0}^{t} \int_{-\infty}^{0} \mu_{J_{y}} p(s, y) d y d s \text { for all } t \in\left[0, T_{\max }\right) .
$$

Finally, using assumptions (8) and (10), we have, for all $y \leq 0$,

$$
\mu_{J_{y}}=\int_{-\infty}^{-y} m J_{y}(m) d m \geq \int_{-\infty}^{0} m J_{y}(m) d m \geq \int_{-\infty}^{0} m \bar{J}(m) d m=\mu .
$$

Thus, since $p$ is nonnegative and $\int_{-\infty}^{0} p(t, m) d m=1$ for all $t \in\left[0, T_{\max }\right)$, we get that $\bar{m}(t) \geq$ $\bar{m}(0)+t U \mu$ for all $t \in\left[0, T_{\max }\right)$. The proof of Lemma 6.5 is thereby complete.

\section{Appendix A: Interpreting condition (29) on $\lim _{z \rightarrow+\infty} z+\omega(z)$}

We here show that the assumption (29) of Proposition 3.7, namely $\lim _{z \rightarrow+\infty}(z+\omega(z)) \in$ $[0,+\infty)$, means that any parent can give mutant offspring with the optimal fitness 0 , that is, 
$\sup \left\{\operatorname{supp} J_{y}\right\}=-y$ for every $y \leq 0$. To do so, differentiating equation (15) with respect to $z$, we have (as in (17)):

$$
\mathcal{C}_{y}^{\prime}(z)=\mathcal{C}_{\star}^{\prime}(z)+\omega^{\prime}(z) y \text { for all } y \leq 0 \text { and } z \geq 0,
$$

where the $\mathcal{C}^{\infty}\left(\mathbb{R}_{+}\right)$functions $\mathcal{C}_{y}$ and $\mathcal{C}_{\star}$ are defined by (16). Now, [17, Lemma 4.5] yields $\lim _{z \rightarrow+\infty} \mathcal{C}_{y}^{\prime}(z)=\sup \left\{\operatorname{supp} J_{y}\right\}$ and $\lim _{z \rightarrow+\infty} \mathcal{C}_{\star}^{\prime}(z)=\sup \left\{\operatorname{supp} J_{0}\right\}$. Passing to the limit $z \rightarrow+\infty$ in $(81)$, it follows that $\lim _{z \rightarrow+\infty} \omega^{\prime}(z)$ exists and

$$
\lim _{z \rightarrow+\infty} \omega^{\prime}(z) y=\sup \left\{\operatorname{supp} J_{y}\right\}-\sup \left\{\operatorname{supp} J_{0}\right\}
$$

for all $y \leq 0$. Assumption (29) then implies that $\omega^{\prime}(z) \rightarrow-1$ as $z \rightarrow+\infty$, hence

$$
-y=\sup \left\{\operatorname{supp} J_{y}\right\}-\sup \left\{\operatorname{supp} J_{0}\right\}
$$

for all $y \leq 0$. On the other hand, assumption (9) yields in particular $\sup \left\{\operatorname{supp} J_{0}\right\} \leq 0$. If $\sup \left\{\operatorname{supp} J_{0}\right\}=-\alpha<0$, then $\sup \left\{\operatorname{supp} J_{y}\right\}=-y-\alpha$ for all $y \leq 0$, contradicting assumption (18) for $y>0$ small. Therefore, $\sup \left\{\operatorname{supp} J_{0}\right\}=0$, and

$$
\sup \left\{\operatorname{supp} J_{y}\right\}=-y
$$

for all $y \leq 0$. In other words, any parent can give mutant offspring with the optimal fitness 0 .

\section{Appendix B: Proof of property (71) on $\omega$}

First of all, by taking $z=0$ in (11), we immediately get that

$$
\omega(0)=0 .
$$

Second, since

$$
\int_{-\infty}^{-y} J_{y}(m) \mathrm{e}^{z m} d m \leq \mathrm{e}^{-z y} \int_{-\infty}^{-y} J_{y}(m) d m
$$

for all $y \leq 0$ and $z \geq 0$, it follows from (8) and (9) that

$$
\int_{-\infty}^{-y} J_{y}(m) \mathrm{e}^{z m} d m \leq \mathrm{e}^{-z y}
$$

for all $y \leq 0$ and $z \geq 0$. Then, using (11), we get that $M_{\star}(z) \mathrm{e}^{\omega(z) y} \leq \mathrm{e}^{-z y}$ and, for every $y<0$, we obtain that $\left(\ln M_{\star}(z)\right) / y+\omega(z) \geq-z$ for all $z \geq 0$. The limit as $y \rightarrow-\infty$ then yields $\omega(z) \geq-z$ for all $z \geq 0$.

Third, observe that assumption (11) yields

$$
\omega(z)=\frac{\mathcal{C}_{y}(z)-\mathcal{C}_{\star}(z)}{y}
$$

for all $y<0$ and $z \geq 0$. Thus, as $\mathcal{C}_{y}$ and $\mathcal{C}_{\star}$ belong to $\mathcal{C}^{\infty}\left(\mathbb{R}_{+}\right)$, the function $\omega$ belongs to $\mathcal{C}^{\infty}\left(\mathbb{R}_{+}\right)$too. In order to show the concavity of $\omega$, it is then sufficient to prove that $\omega^{\prime \prime} \leq 0$ in $\mathbb{R}_{+}$. But since $\mathcal{C}_{y}$ is convex in $\mathbb{R}_{+}$for every $y \leq 0$, the previous displayed formula implies that

$$
\omega^{\prime \prime}(z)=\frac{\mathcal{C}_{y}^{\prime \prime}(z)-\mathcal{C}_{\star}^{\prime \prime}(z)}{y} \leq-\frac{\mathcal{C}_{\star}^{\prime \prime}(z)}{y}
$$

for all $y<0$ and $z \geq 0$. The limit as $y \rightarrow-\infty$ yields $\omega^{\prime \prime}(z) \leq 0$ for all $z \geq 0$, and the proof of $(71)$ is thereby complete. 


\section{References}

[1] M Alfaro and R Carles. Explicit solutions for replicator-mutator equations: Extinction versus acceleration. SIAM J. Appl. Math., 74(6):1919-1934, 2014.

[2] M Alfaro and R Carles. Replicator-mutator equations with quadratic fitness. Proc. Amer. Math. Soc., 145:5315-5327, 2017.

[3] M Alfaro and J Coville. Propagation phenomena in monostable integro-differential equations: Acceleration or not? J. Diff. Equations, 263(9):5727-5758, 2017.

[4] M Alfaro, J Coville, and G Raoul. Bistable travelling waves for nonlocal reaction diffusion equations. Discrete Contin. Dyn. Syst., 34:1775-1791, 2014.

[5] M Alfaro and M Veruete. Evolutionary branching via replicator-mutator equations. arXiv preprint arXiv:1802.00501, 2018.

[6] H Berestycki, G Nadin, B Perthame, and L Ryzhik. The non-local Fisher-KPP equation: travelling waves and steady states. Nonlinearity, 22(12):2813-2844, 2009.

[7] A Carr and J Chmaj. Uniqueness of travelling waves for nonlocal monostable equations. Proc. Amer. Math. Soc., 132(8):2433-2439, 2004.

[8] C Cortázar, J Coville, M Elgueta, and S Martínez. A nonlocal inhomogeneous dispersal process. J. Diff. Equations, 241(2):332-358, 2007.

[9] A Couce and O A Tenaillon. The rule of declining adaptability in microbial evolution experiments. Frontiers in Genetics, 6:99, 2015.

[10] J Coville. Singular measure as principal eigenfunction of some nonlocal operators. Appl. Math. Lett., 26(8):831-835, 2013.

[11] J Coville and L Dupaigne. On a nonlocal equation arising in population dynamics. Proc. Roy. Soc. Edinburgh Sect. A, 137:1-29, 2007.

[12] J Fang and X-Q Zhao. Monotone wavefronts of the nonlocal Fisher-KPP equation. Nonlinearity, 24:3043-3054, 2011.

[13] G Faye and M Holzer. Modulated traveling fronts for a nonlocal Fisher-KPP equation: a dynamical systems approach. J. Diff. Equations, 258(7):2257-2289, 2015.

[14] J Garnier. Accelerating solutions in integro-differential equations. SIAM J. Math. Anal., 43:1955-1974, 2011.

[15] J Garnier, F Hamel, and L Roques. Transition fronts and stretching phenomena for a general class of reaction-dispersion equations. Discrete Contin. Dyn. Syst., 37:743-756, 2017.

[16] S Genieys, V Volpert, and V Auger. Pattern and waves for a model in population dynamics with nonlocal consumption of resources. Math. Model. Nat. Phenom., 1:63-80, 2006.

[17] M-E Gil, F Hamel, G Martin, and L Roques. Mathematical properties of a class of integrodifferential models from population genetics. SIAM J. Appl. Math., 77:1536-1561, 2017. 
[18] B H Good and M M Desai. The impact of macroscopic epistasis on long-term evolutionary dynamics. Genetics, 199:177-190, 2015.

[19] S A Gourley. Travelling front solutions of a nonlocal fisher equation. J. Math. Biol., 41:272-284, 2000.

[20] Q Griette. Singular measure traveling waves in an epidemiological model with continuous phenotypes. arXiv preprint arXiv:1710.02240, 2017.

[21] F Hamel and L Ryzhik. On the nonlocal Fisher-KPP equation: steady states, spreading speed and global bounds. Nonlinearity, 27(11):2735-2753, 2014.

[22] M Kimura. A stochastic model concerning the maintenance of genetic variability in quantitative characters. Proc. Natl. Acad. Sci. USA, 54(3):731-736, 1965.

[23] M Kirkpatrick and N H Barton. Evolution of a species' range. Am. Nat., 150:1-23, 1997.

[24] S Kryazhimskiy, D P Rice, E R Jerison, and M M Desai. Global epistasis makes adaptation predictable despite sequence-level stochasticity. Science, 344(6191):1519-1522, 2014.

[25] A Lambert. Population dynamics and random genealogies. Stoch. Models, 24(S1):45-163, 2008.

[26] R Lande. The genetic covariance between characters maintained by pleiotropic mutations. Genetics, 94(1):203-215, 1980.

[27] M Lässig, V Mustonen, and A M Walczak. Predicting evolution. Nat. Ecol. Evol., 1(3):77, 2017.

[28] G Martin, S F Elena, and T Lenormand. Distributions of epistasis in microbes fit predictions from a fitness landscape model. Nature Genetics, 39(4):555-560, 2007.

[29] G Martin and L Roques. The non-stationary dynamics of fitness distributions: Asexual model with epistasis and standing variation. Genetics, 204:1541-1558, 2016.

[30] M H Protter and H F Weinberger. Maximum Principles in Differential Equations. Prentice-Hall, Inc., Englewood Cliffs, N.J., 1967.

[31] L Roques, J Garnier, and G Martin. Beneficial mutation-selection dynamics in finite asexual populations: a free boundary approach. Sci. Rep., 7(1):17838, 2017.

[32] K Schumacher. Travelling-front solutions for integro-differential equations. I. J. Reine Angew. Math., 316:54-70, 1980.

[33] P D Sniegowski and P J Gerrish. Beneficial mutations and the dynamics of adaptation in asexual populations. Philos. Trans. Royal Soc. B, 365(1544):1255-1263, 2010.

[34] O A Tenaillon. The utility of Fisher's geometric model in evolutionary genetics. Annual Review of Ecology, Evolution, and Systematics, 45(1):179-201, 2014.

[35] L S Tsimring, H Levine, and D A Kessler. RNA virus evolution via a fitness-space model. Phys. Rev. Lett., 76(23):4440, 1996. 
[36] J Y Wakano and Y Iwasa. Evolutionary branching in a finite population: deterministic branching vs. stochastic branching. Genetics, 193(1):229-241, 2013.

[37] D Waxman and J R Peck. Pleiotropy and the preservation of perfection. Science, 279(5354):1210-1213, 1998.

[38] D Waxman and J R Peck. The frequency of the perfect genotype in a population subject to pleiotropic mutation. Theoretical Population Biology, 69(4):409-418, 2006.

[39] H F Weinberger. Long-time behavior of a class of biological models. SIAM J. Math. Anal., 13(3):353-396, 1982.

[40] H F Weinberger. On spreading speeds and traveling waves for growth and migration in periodic habitat. J. Math. Biol., 45:511-548, 2002.

[41] $\mathrm{H}$ Yagisita. Existence and nonexistence of travelling waves for a nonlocal monostable equation. Publ. RIMS, 45:925-953, 2009. 